\title{
Minimum momentum flux ratio required to prevent air curtain breakthrough in case of cross-curtain pressure gradients: CFD versus analytical equation
}

\author{
Adelya Khayrullina ${ }^{1}(\varangle)$, Twan van Hooff ${ }^{1,2}$, Claudio Alanis Ruiz², Bert Blocken ${ }^{1,2}$, GertJan van Heijst ${ }^{3}$ \\ 1. Department of the Built Environment, Eindhoven University of Technology, Eindhoven, The Netherlands \\ 2. Department of Civil Engineering, KU Leuven, Belgium \\ 3. Department of Applied Physics, Eindhoven University of Technology, Eindhoven, The Netherlands
}

\section{Abstract}

This paper presents a numerical study on the required momentum flux ratio to prevent air curtain breakthrough in case of cross-curtain (i.e. cross-jet) pressure gradients. 2D steady Reynolds-averaged Navier-Stokes (RANS) CFD simulations with the RNG $k-\varepsilon$ turbulence model are employed for jet Reynolds numbers ranging from 5,000 to 30,000 . First, the computational model is validated based on particle image velocimetry (PIV) measurements. Second, the influence of several jet parameters on the separation efficiency is evaluated for a moderate cross-jet pressure difference of $10 \mathrm{~Pa}$. These are the ratio of the jet discharge momentum flux to the jet cross-flow momentum flux (momentum flux ratio), the jet height-to-width ratio and the jet discharge angle. Finally, the minimum deflection modulus to prevent jet breakthrough and the corresponding momentum flux ratio by an analytical equation and by CFD are compared. The results show that, for the configuration under study: (1) jets with the smallest height-to-width ratios $(\beta=18)$ provide the highest separation efficiency; (2) inclined jets with discharge angles $a_{0}=5^{\circ}$ and $10^{\circ}$ provide slightly higher separation efficiency than straight jets $\left(a_{0}=0^{\circ}\right)$ and jets with $a_{0}=20^{\circ} ;$ (3) the maximum modified separation efficiency is reached at lower momentum flux ratios for jets with smaller height-to-width ratios and for inclined jets; (4) the analytical and CFD values of the optimal momentum flux ratio differ with up to $31.2 \%$. This study shows how the separation efficiency of air curtains can be improved by adjusting certain jet parameters.
\end{abstract}

\section{Keywords}

plane turbulent jet, air curtain, separation efficiency, CFD

\section{Article History}

Received: 27 September 2019

Revised: 05 February 2020

Accepted: 02 March 2020

(c) The Author(s) 2020

\section{Introduction}

Air curtains (ACs) are plane turbulent impinging jets (PTIJs) at moderate to high Reynolds numbers that are used to separate two environments in terms of heat and mass transfer. ACs are applied in many practical applications, for example, at entrances of buildings and refrigerated rooms to reduce heat losses (e.g. Wang and Zhong 2014; Goubran et al. 2016; Gonçalves et al. 2019; Yang et al. 2019) and in laboratories or operating theatres (e.g. Nino et al. 2011; Chen et al. 2013; Zhai and Osborne 2013) to reduce contamination hazard. The existing literature on PTIJs and ACs can be divided in two clear categories: (1) basic studies on PTIJs; and (2) application-oriented studies on ACs, both of which can be performed experimentally and numerically (e.g. using computational fluid dynamics (CFD)). Note that an extensive literature review on the topic of $\mathrm{AC}$ is not the purpose of this paper and only some relevant studies are discussed below.

With regard to basic studies, a non-exhaustive overview of experimental studies on PTIJs up to 2016 is provided in Khayrullina et al. (2017). These experimental studies highlighted the existence of vortical structures in the jet flow, their role in the dissipation of jet energy to the ambient environment, and the existence of $3 \mathrm{D}$ vortices in the impingement region, in which thermal energy can be exchanged between the jet and the impingement surface. A non-exhaustive overview of numerical studies on PTIJs

E-mail: a.khayrullina@tue.nl 


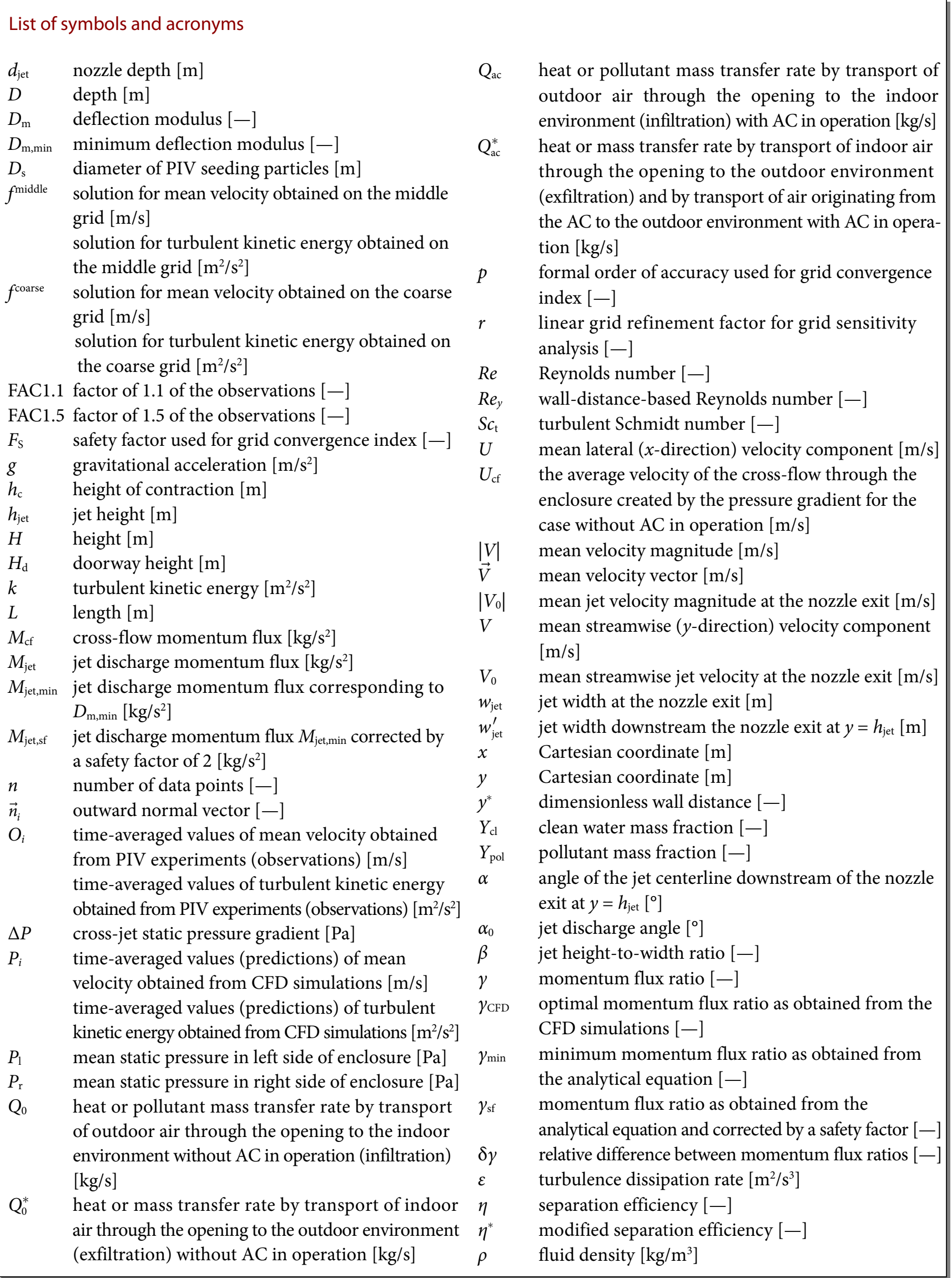




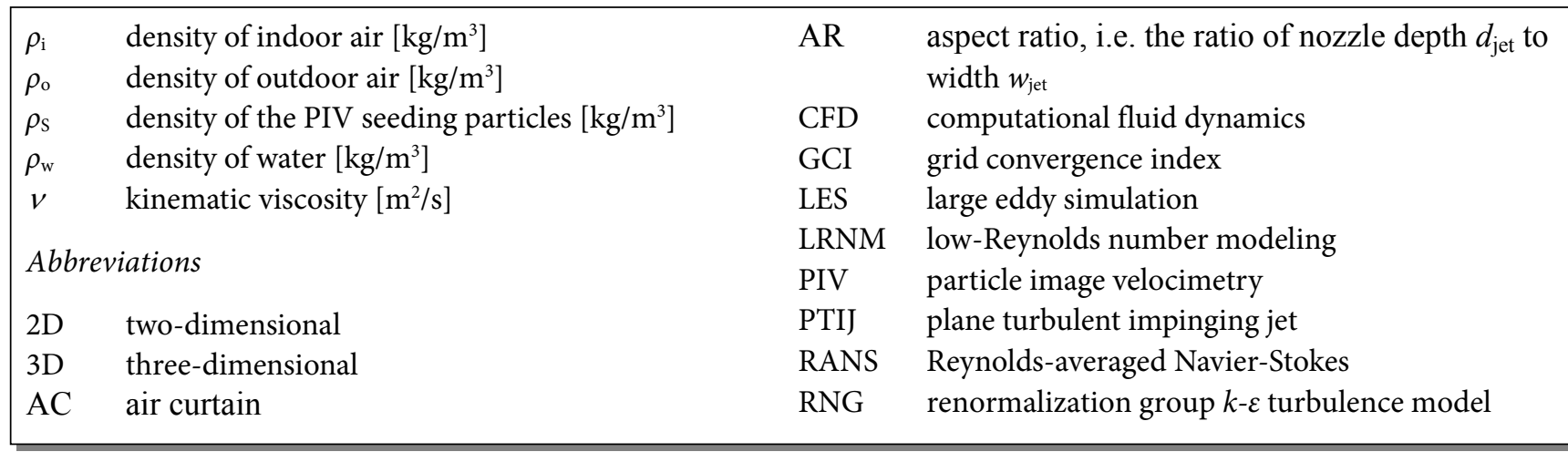

using steady RANS is provided in Khayrullina et al. (2019). This overview concluded that the majority of the numerical studies on PTIJ only focused on heat transfer within the jet impingement region. In line with commonly used general guidelines for CFD studies in building simulation (e.g. Casey and Wintergerste 2000; Nielsen et al. 2007; Blocken 2015), several numerical studies on PTIJs indicated the importance of grid-independent results (e.g. Jaramillo et al. 2008; Rhea et al. 2009), the use of at least second-order discretization schemes (e.g. Craft et al. 1993; Park et al. 2003; Dutta et al. 2013) and the use of low-Reynolds number modeling (LRNM) as a near-wall modeling approach (e.g. Isman et al. 2008). Furthermore, Isman et al. (2008), Jaramillo et al. (2008), Rhea et al. (2009), Kozeoglu and Baskaya (2010) and others highlighted the importance of accurately reproducing the geometry of the jet nozzle and representative boundary conditions of the nozzle exit in CFD simulations of air curtains.

Application-oriented studies generally focused on the dimensioning of the $\mathrm{AC}$, determining the $\mathrm{AC}$ efficiency by empirical formulae, and defining the most influential parameters with respect to the AC efficiency. The efficiency of ACs $(\eta)$ can be defined based on the rate of heat or mass transfer through the opening with an $\mathrm{AC}$ compared to that of the same opening without an AC (e.g. Frank and Linden 2014; Gonçalves et al. 2019):

$\eta=1-\frac{Q_{\mathrm{ac}}}{Q_{0}}$

with $Q_{\mathrm{ac}}$ the heat or mass transfer rate by transport of outdoor air through an opening with $\mathrm{AC}$ to the indoor environment (infiltration), and $Q_{0}$ the heat or mass transfer rate by transport of outdoor air through an opening without AC to the indoor environment. It is important to provide aerodynamic sealing along the entire height and width of the opening between the two environments separated by the AC, while also limiting excessive mixing between the jet and the ambient air. Alanis Ruiz et al. (2018) expanded the definition of separation efficiency to the modified separation efficiency $\left(\eta^{*}\right)$ that includes mass transfer due to both infiltration and exfiltration and due to the airflow from the AC:

$\eta^{*}=1-\frac{Q_{\mathrm{ac}}+Q_{\mathrm{ac}}^{*}}{Q_{0}+Q_{0}^{*}}$

with $Q_{\mathrm{ac}}^{*}$ the heat or mass transfer rate with $\mathrm{AC}$, calculated as the sum of heat or mass transfer rate by transport of indoor air through the opening with an $\mathrm{AC}$ to the outdoor environment (exfiltration), and the heat or mass transfer rate by the transport of air originating from the $\mathrm{AC}$ to the outdoor environment; and $Q_{0}^{*}$ the heat or mass transfer rate by transport of indoor air through the opening without an $\mathrm{AC}$ to the outdoor environment (exfiltration).

Along with environmental parameters, e.g. temperature and pressure differences between the two environments, the separation efficiency of an AC depends on a wide range of jet parameters. The jet nozzle shape has a direct impact on the profiles of jet velocity and turbulence intensity when the jet exits from the nozzle. For example, Deo et al. (2007a) showed that plane free jets with sharp-edged nozzles result in a higher jet spreading rate than those from smoothlyshaped nozzles, which can negatively influence the AC separation efficiency. This was explained by the higher turbulence intensity levels at the nozzle exit and the faster shear layer growth compared to jets issued by a smoothlyedged nozzle. To the best knowledge of the authors, no studies on ACs are available for different nozzle shapes, although, conclusions of studies on plane free jets (e.g. Deo et al. 2007a) could be applied to ACs as well, as long as the other jet parameters in those studies, such as jet aspect ratio and Reynolds number, are similar to those of ACs. The jet aspect ratio ( $A R$; ratio of nozzle depth to width at the nozzle exit $d_{\text {jet }} / w_{\text {jet }}$, where $\left.w_{\text {jet }}<d_{\text {jet }}\right)$ is another important parameter with respect to the jet flow and thus the separation efficiency. Deo et al. (2007b) found that jets at certain $R e$ and smaller $A R$ experience a stronger influence of the sidewalls on the jet flow near the nozzle exit, which enhances velocity fluctuations and results in higher turbulence 
intensities and higher entrainment rates of ambient fluid compared to jets with larger $A R$. On the other hand, they reported that jets with larger $A R$ have higher jet spreading and jet decay rates in the jet far field (jet intermediate region). Finally, the jet height-to-width ratio ( $\beta$, ratio of the distance from the nozzle exit to the impingement plate to nozzle width $\left.h_{\text {jet }} / w_{\text {jet }}\right)$ was studied by - among others - Shih et al. (2011) and Moureh and Yataghene (2016), who showed that for increasing height-to-width ratio the AC separation efficiency decreases. Jets with increased height-to-width ratio have a shorter potential core length in relation to $h_{\text {jet }}$ and experience larger entrainment of the ambient fluid compared to a jet with a smaller height-to-width ratio.

Several studies (e.g. Hayes and Stoecker 1969a; Sirén 2003; Frank and Linden 2014) specified the minimum ratio of jet momentum flux to cross-jet forces acting on AC to ensure the optimal separation efficiency for a doorway. At a certain jet velocity the separation efficiency $\eta$ (Eq. (1)) of the jet reaches its maximum value. With further increase of the jet velocity the separation efficiency of the air curtain decreases, which is caused by increased mixing with the ambient fluid along the jet and near the floor due to the higher discharge momentum flux of the jet. The jet turbulence intensity also has an effect on jet spreading and entrainment rate. Hayes and Stoecker (1969b) recommended to reduce the turbulence intensity at the nozzle exit to decrease the entrainment rate of the ambient fluid into the jet. The turbulence intensity can be reduced by equipping the discharge nozzle with fine mesh screens and by avoiding sharp-edged nozzles. The separation efficiency of the jet can also be increased by inclining the jet to the exterior environment, as this counterbalances pressure differences across the doorway and as often the outdoor static pressure is higher than the indoor static pressure (Hayes and Stoecker 1969b; Costa et al. 2006; Valkeapää et al. 2006). The defined optimal jet discharge angles reported in the literature vary from $15^{\circ}$ (Costa et al. 2006) to $30^{\circ}$ (Hayes and Stoecker 1969b).

Hayes and Stoecker (1969a) introduced the so-called "deflection modulus" to determine the minimum jet momentum flux required for sealing a doorway with an air curtain and preventing breakthrough of the jet. Jet breakthrough occurs if the jet bends at a distance from the impingement plate (floor) and does not impinge on the floor due to insufficient jet momentum flux to counteract a pressure gradient across the doorway (e.g. Hayes and Stoecker 1969a; Frank and Linden 2014). Subsequently, unwanted heat and/or mass transfer occurs through the lowest part of the opening (below the bended jet) between the two environments that needed to be aerodynamically separated. The deflection modulus is defined as the ratio of the momentum flux of the air curtain jet at the nozzle exit to the transverse forces acting across the jet due to stack pressure across the doorway. Based on the conservation of momentum, the authors provided an equation to estimate the required minimum value of the deflection modulus $\left(D_{\mathrm{m}, \min }\right)$ of an air curtain at the doorway of a heated room:

$D_{\text {m,min }}=\frac{\rho w_{\text {jet }}\left|V_{0}\right|^{2}}{g H_{\mathrm{d}}^{2}\left(\rho_{\mathrm{o}}-\rho_{\mathrm{i}}\right)}$

with $\rho$ the density of the jet at the nozzle exit, $w_{\text {jet }}$ the jet width at the nozzle exit, $g$ the gravitational acceleration $(\approx$ $\left.9.8 \mathrm{~m} / \mathrm{s}^{2}\right),\left|V_{0}\right|$ the jet velocity magnitude (i.e. the magnitude of the $3 \mathrm{D}$ velocity vector) at the nozzle exit, $H_{\mathrm{d}}$ the height of the doorway, and $\rho_{\mathrm{o}}$ and $\rho_{\mathrm{i}}$ the density of air outside and inside the room, respectively. Moreover, it was suggested to multiply the velocity at the nozzle exit by a safety factor of 1.3 to 2.0 (Hayes and Stoecker 1969b), resulting in a safety factor for the deflection modulus between 1.7 to 4.0, in order to ensure a stable air curtain in real situations.

Recently some experimental and numerical studies were performed that used the deflection modulus to estimate the optimal jet momentum flux. The CFD study of Costa et al. (2006) considered an air curtain restricting heat transfer between two environments at different temperatures. They showed that a deflection modulus $D_{\text {m,min }}$ multiplied with a safety factor of approximately 2.6 prevented air curtains from breakthrough. Foster et al. (2006) performed a CFD study of an air curtain installed in a doorway of a building and showed that a safety factor of 1.6 applied to $D_{\mathrm{m}, \mathrm{min}}$ provided the highest separation efficiency. Van Belleghem et al. (2012) conducted a CFD study of an air curtain flow in a sealed cold room application. They showed that a safety factor of 2 was required for the deflection modulus $\left(D_{\mathrm{m}, \min }\right)$ to be able to provide a stable air curtain without breakthrough, which is in line with the initial recommendation by Hayes and Stoecker (1969b). Frank and Linden (2014) investigated the performance of an air curtain in a doorway of a ventilated building both with analytical and experimental models. They considered a number of situations with different positions of the neutral pressure level height, which is the height at which indoor and outdoor pressures are equal. For situations where the neutral pressure level height was located slightly above the mid-height of the door opening, the defined $D_{\mathrm{m} \text {,min }}$ had to be multiplied by a safety factor of 2.8 to prevent breakthrough. However, a safety factor of 7 had to be applied in case the neutral pressure level height was far above the mid-height of the door opening. This higher safety factor can be explained by the ventilation losses through the additional top opening introduced by Frank and Linden (2014), whereas the study of Hayes and Stoecker (1969b) considered only transverse forces acting on the doorway. 
From the literature it can be concluded that application studies on AC are often very case-specific and in spite of their value to solve specific real-life problems, lack generality in their conclusions. On the other hand, basic and generic studies on PTIJs generally focused on heat transfer within the jet impingement region. As a result, there is a scarcity with respect to detailed generic studies on ACs and PTIJs that assess the influence of the jet parameters on the separation efficiency. The primary focus of this paper is therefore (1) to provide a parametric analysis of the impact of several jet parameters (momentum flux ratio, jet heightto-width ratio, jet discharge angle) on jet separation efficiency; and (2) to perform a comparison between values of the momentum flux ratio based on $D_{\mathrm{m}, \min }$ as obtained from the analytical equation by Hayes and Stoecker (1969b) and values of the momentum flux ratio required for a maximum modified separation efficiency as obtained from CFD simulations, and this for a wide range of parameters. Note that the jet discharge angle $\alpha_{0}$ is defined as the jet angle at the nozzle exit $(y=0 \mathrm{~m})$. This paper focuses on the generic situation of an isothermal AC separating two environments subjected to a moderate cross-jet pressure difference (in this study fixed at $10 \mathrm{~Pa}$ ). 2D steady RANS CFD simulations are performed for jet Reynolds numbers ranging from 5,000 to 30,000 . First, the computational model is validated based on particle image velocimetry (PIV) data by Khayrullina et al. (2017). Subsequently, a parametric study is performed, in which the influence of several jet parameters on jet separation efficiency is evaluated. Finally, for each jet configuration (depending on jet heightto-width ratio and jet discharge angle), the ratio of jet discharge momentum flux to jet cross-flow momentum flux (momentum flux ratio) at which the modified separation efficiency of AC reaches its maximum value is obtained. These values are compared with the values of the momentum flux ratio obtained from the deflection modulus as defined by Hayes and Stoecker (1969b). The paper is structured as follows. Section 2 presents the experimental set-up, the PIV measurements, the computational model and the computational settings/parameters used in the CFD simulations. Finally, it presents the results of the validation study. Section 3 presents the computational settings and parameters for the case study. Section 4 presents the results of the case study. Finally, Sections 5 (Discussion) and Section 6 (Conclusions) conclude this paper.

\section{Validation study}

\subsection{Reduced-scale PIV measurements}

The PIV measurements of PTIJ flow were conducted in a water channel with dimensions $L \times H \times D=2.00 \mathrm{~m} \times$
$0.36 \mathrm{~m} \times 0.30 \mathrm{~m}$. A reduced-scale model was considered to allow the full PTIJ to be captured within at most two field of views in the PIV measurements. To ensure dynamic similarity, the Reynolds number in the reduced-scale setup should be the same as that in the full-scale equivalent. Therefore, the reduced-scale experiments were performed with water as working fluid and at a geometric scale of 1:8. Because the ratio of the kinematic viscosities of air and water is equal to 15 at $20^{\circ} \mathrm{C}$, the inlet velocity in the reduced-scale setup was taken as $8 / 15$ times that of the full-scale equivalent. The size of the water channel represented an actual air curtain jet in full scale of $H \times D=2.88 \mathrm{~m} \times 2.40 \mathrm{~m}$. The experimental setup is shown in Fig. 1. A water column (not shown in the figure) created hydrostatic pressure and drove the flow through a conditioning section consisting of one honeycomb and two screens in order to provide a uniform flow and to reduce the turbulence intensity. Subsequently, a plane jet was issued vertically from the smoothly-shaped nozzle. In the experiments, the mean streamwise velocity at the nozzle exit $V_{0}$ was equal to $0.5 \mathrm{~m} / \mathrm{s}$, corresponding to $R e=8,000$. The Reynolds number was defined as $R e=$ $\left(V_{0} w_{\text {jet }}\right) / v$, with $w_{\text {jet }}=16 \mathrm{~mm}$ the jet width at the nozzle exit and $v$ the kinematic viscosity at $20^{\circ} \mathrm{C}$. The jet height $\left(h_{\text {jet; }}\right.$ distance from the nozzle exit to the impingement plate) was $360 \mathrm{~mm}$ and the nozzle depth (dimension in spanwise direction; $z$-direction) was $300 \mathrm{~mm}$. The nozzle aspect ratio was $A R=18.75$ and the jet height-to-width ratio was $\beta=22.5$.

The PIV measurements were conducted with a 2D PIV system consisting of a solid-state frequency-doubled Nd:Yag laser (wavelength $532 \mathrm{~nm}$ and repetition rate $15 \mathrm{~Hz}$ ) as an illuminating source and a charge coupled device (CCD) camera $(1600 \times 1200$ pixels resolution, up to 30 frames/s $)$ for image acquisition. Seeding was provided by polyamide particles $\left(D_{\mathrm{S}}=50 \mu \mathrm{m}\right.$, density $\left.\rho_{\mathrm{S}}=1,030 \mathrm{~kg} / \mathrm{m}^{3}\right)$ added to the water. The particles were illuminated by means of a

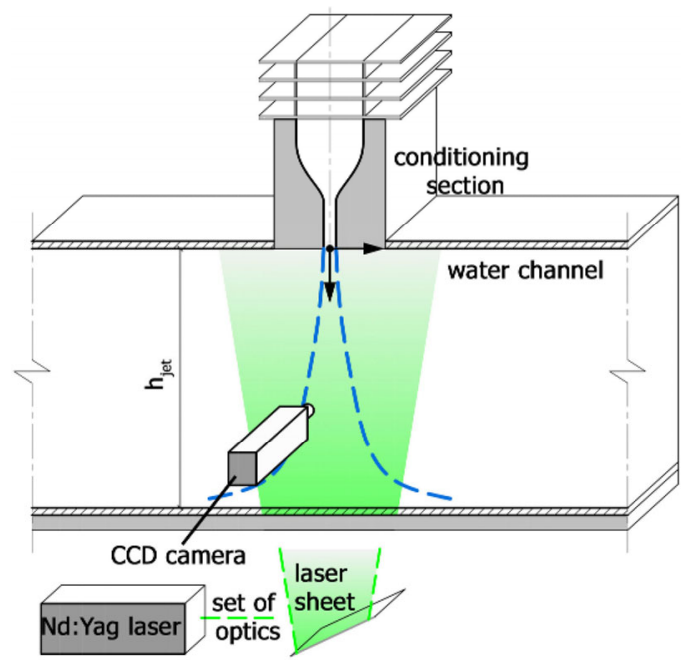

Fig. 1 Experimental set-up (adapted from Khayrullina et al. 2017) 
light sheet delivered at the bottom of the water channel. The PIV measurements provided information on the mean velocities and turbulence intensities in the vertical centerplane. More information on the experimental set-up and measurement results can be found in Khayrullina et al. (2017). The experimental results will be shown together with the CFD results in the following subsections.

\subsection{Computational model}

\subsubsection{Computational geometry and grid}

The $2 \mathrm{D}$ computational geometry $\left(L \times\left(h_{\text {jet }}+h_{\mathrm{c}}\right)\right)=(2.0 \times$ $(0.36+0.15)) \mathrm{m}^{2}$ replicates the experimental setup described in Section 2.1 (Fig. 2). A 2D instead of a 3D geometry is used to limit the computational costs for the parametric study. The simulation results for a $2 \mathrm{D}$ geometry have been compared to the results for a 3D geometry and only negligible differences have been observed between their results in the vertical centerplane, which can be explained by the limited influence of sidewalls at a jet aspect ratio of $A R=18.75$. The maximum difference between the results obtained along the jet centerline by $2 \mathrm{D}$ and $3 \mathrm{D}$ CFD simulations is less than $7 \%$ for predictions of mean velocity and less than $5 \%$ for predictions of turbulent kinetic energy.

A structured grid with a high spatial resolution is applied within the smoothly-shaped nozzle, within the zone outlined by the jet spreading rate and within the jet impingement zone. For the remainder of the computational domain an unstructured quadrilateral mesh is applied. The grid resolution is determined by means of a grid sensitivity analysis using three different grids (see Section 2.3 for results) with a grid-refinement factor of $\sqrt{2}$ in each direction: coarse grid (23,553 cells), middle grid (41,209 cells) and fine grid

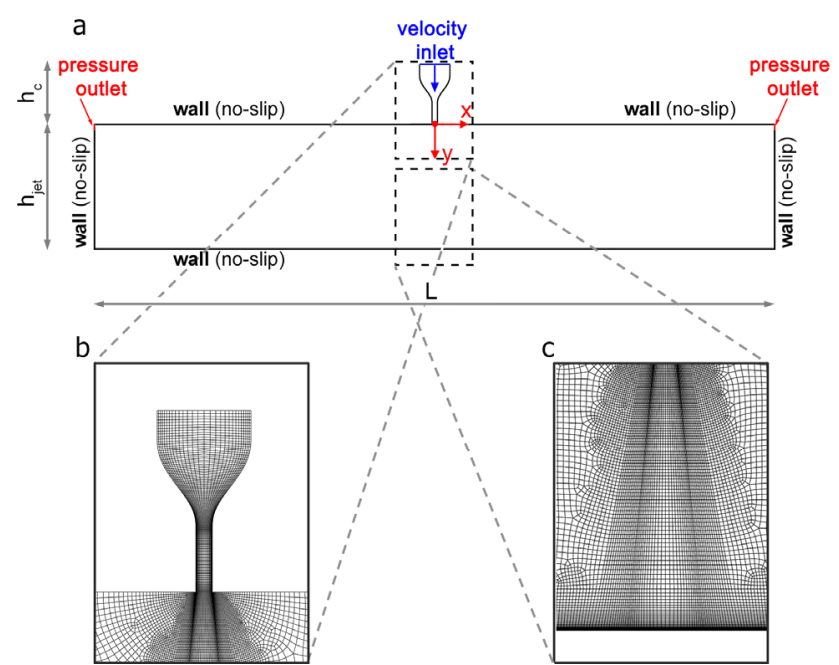

Fig. 2 (a) 2D computational geometry with indication of boundary conditions. (b-c) Computational grid at: (b) smoothly-shaped nozzle; (c) impingement plate
(85,110 cells). Across the computational inlet, i.e. the inlet of the nozzle, 28, 40 and 56 cells are used for the coarse, middle and fine grids, respectively (Fig. 3). The dimensionless wall distances ( $y^{*}$, average values) for the coarse, middle and fine grids within the smoothly-shaped jet nozzle are equal to 4, 2.5 and 1, respectively, while at the impingement plate the average $y^{*}$ values are 5, 1.5 and 0.5 , respectively. These three grids with $y^{*}<5$ at the walls enable lowReynolds number modeling for the wall-adjacent flow, i.e. resolving this flow all the way down the viscous sublayer, rather than resorting to wall function modeling.

\subsubsection{Boundary conditions}

At the inlet of the computational domain a uniform velocity in vertical direction corresponding to $V_{0, \mathrm{CFD} \text {, inlet }}=0.085 \mathrm{~m} / \mathrm{s}$ is imposed, which results in $V_{0}=0.5 \mathrm{~m} / \mathrm{s}$ and $R e=8,000$ at the nozzle exit. The turbulence intensity at the domain inlet is set to $15 \%$, which results in a turbulence intensity on the jet centerline near the nozzle exit corresponding to the measured value $(=4 \%)$. At the outlets zero static gauge pressure is applied. The remaining surfaces are modeled as no-slip walls.

\subsubsection{Turbulence model and solver settings}

The 2D steady RANS equations are solved with the commercial CFD code ANSYS Fluent 16 (ANSYS 2013) using the renormalization group (RNG) $k-\varepsilon$ turbulence model (Yakhot et al. 1992) to provide closure to the governing equations. This model is chosen due to its reported good overall performance in previous PTIJ studies (e.g. Isman et al. 2008; Sharif and Mothe 2009; Khayrullina et al. 2019). Low-Reynolds number modeling (LRNM) is used to solve the near-wall flow. The LRNM option used here consists of the two-layer model. The domain is divided in a
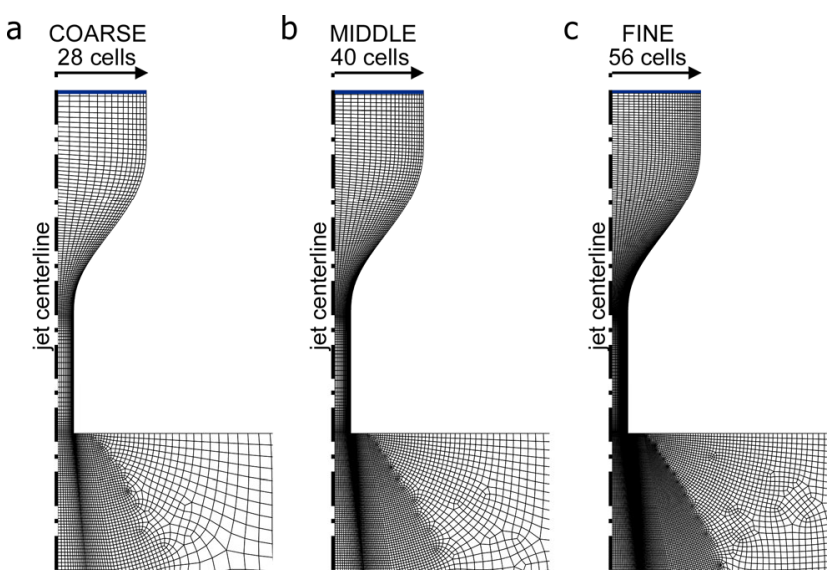

Fig. 3 Computational grid in and near the nozzle with indication of number of cells over the width of the nozzle: (a) coarse grid (23,553 cells); (b) middle grid (41,209 cells); (c) fine grid $(85,110$ cells) 
viscosity-affected region and a fully turbulent region, based on the wall-distance-based Reynolds number $R e_{y}$. The border between both regions is defined as $R e_{y}=200$; if $R e_{y}<200$, the Wolfshtein model is used (Wolfshtein 1969), while for $R e_{y}>200$ the original transport equations of the RNG model are solved. An Eulerian advection-diffusion equation is used to model pollutant dispersion, with the turbulent Schmidt number taken equal to $S \mathcal{c}_{\mathrm{t}}=0.7$ (Tominaga and Stathopoulos 2007). Second-order discretization schemes are used for both the convective and viscous terms of the governing equations and for the turbulence model equations, pressure-velocity coupling is performed by the coupled algorithm, and pressure interpolation is second order. Convergence is considered to be achieved when the scaled residuals (ANSYS 2013) reach the following minimum values: $10^{-14}$ for $x$ and $y$ velocity, $10^{-13}$ for $k$ and $\varepsilon$, and $10^{-12}$ for continuity.

\subsection{Grid sensitivity analysis}

The grid sensitivity analysis is conducted using a coarse, middle and fine grid (see Section 2.2.1). The results of the grid sensitivity analysis in terms of the centerline profiles $\left(x / w_{\text {jet }}=0\right)$ of normalized jet velocity in the vertical direction $\left(V / V_{0}\right)$ and normalized turbulent kinetic energy $\left(k / V_{0}^{2}\right)$ are shown in Fig. 4. The grid-convergence index (GCI) by Roache $(1994,1997)$ is calculated for the coarse grid, which is superimposed on the results for the coarse grid by shaded bounds in Fig. 4, using:

$G C I_{\text {coarse }}=F_{\mathrm{S}}\left|\frac{r^{p}\left(f^{\text {coarse }}-f^{\text {middle }}\right)}{1-r^{p}}\right|$

a

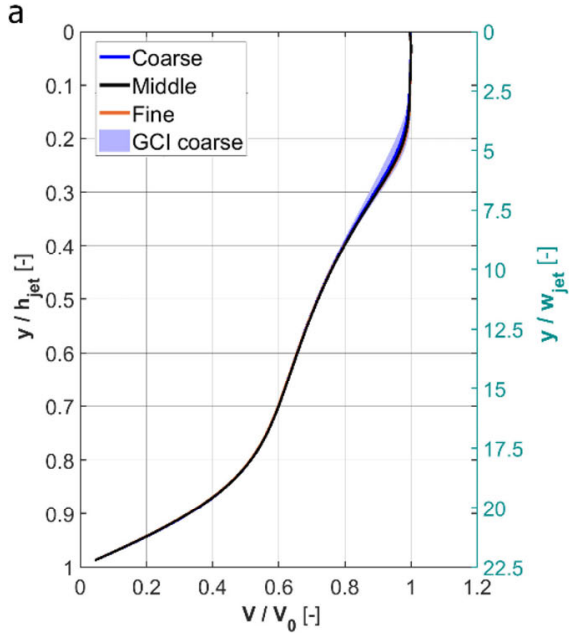

with $F_{\mathrm{S}}$ the safety factor that is equal to the recommended value of 1.25 when at least three grids are analyzed, $r$ the linear grid refinement factor equal to $\sqrt{2}, p$ the formal order of accuracy, which is assigned the value of 2 as second-order discretization schemes are used for the simulations (Roache 1997) and $f^{\text {coarse }}$ and $f^{\text {middle }}$ the solutions obtained on the coarse and middle grid, respectively, which correspond to $V / V_{0}$ and $k / V_{0}^{2}$. The average values of $G C I_{\text {coarse }}$ along the jet centerline are $1 \%$ and $8 \%$ for distributions of mean velocity and turbulent kinetic energy, respectively. The largest discrepancy occurs at the transition between the potential and intermediate jet regions $\left(y / h_{\text {jet }} \approx 0.2\right)$. This is due to the increasing size of the cells further downstream from the nozzle exit along the jet height in combination with the large gradients of mean velocity and turbulent kinetic energy that occur around $y / h_{\text {jet }} \approx 0.2$. Based on this analysis it is concluded that the coarse grid provides nearly grid-independent results and it is therefore used in the remainder of this study.

\subsection{Validation}

Figure 5 shows profiles of $V / V_{0}$ and $k / V_{0}^{2}$ along the jet centerline obtained from the PIV measurements and the CFD simulations with the RNG $k-\varepsilon$ turbulence model. In general, a very good agreement is observed for $V / V_{0}$, while an overestimation of $k / V_{0}^{2}$ in CFD is present along the entire vertical centerline. RNG utilizes a simplified production term of turbulent kinetic energy based on the Boussinesq relationship with the assumption that the turbulence viscosity is an isotropic scalar quantity, which is expected to result in the deviations observed in Fig. 5b.

In order to provide a quantitative assessment of the

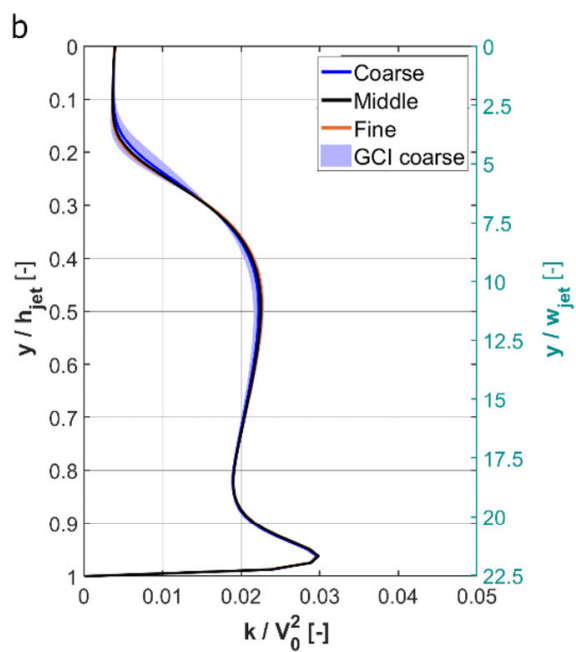

Fig. 4 Results of grid-sensitivity analysis along jet centerline: (a) normalized velocity ( $\left.V / V_{0}\right)$; (b) normalized turbulent kinetic energy $\left(k / V_{0}^{2}\right)$. Blue bounds indicate the GCI index calculated for the coarse grid, $y / w_{\text {jet }}=0$ corresponds to the jet nozzle exit. Note that the three lines almost overlap in both graphs and are therefore less visible 

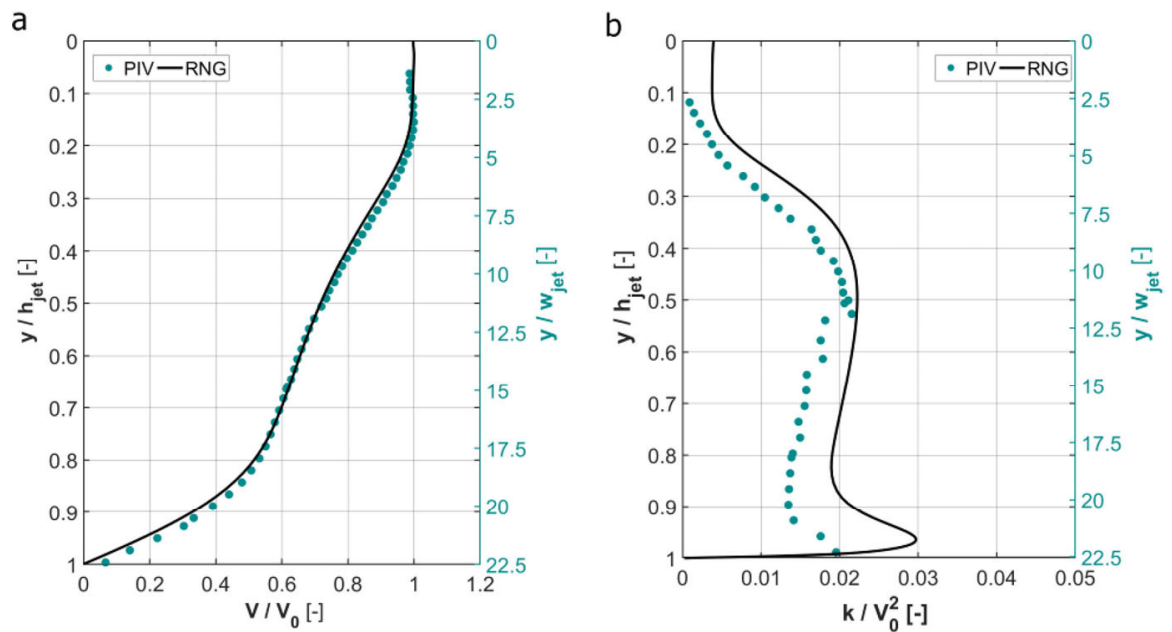

Fig. 5 Comparison of time-averaged PIV results with results of steady RANS CFD simulations obtained along the jet centerline $\left(x / w_{\text {jet }}=0\right)$ : (a) $V / V_{0}$; (b) $k / V_{0}^{2}$

agreement between experimental and numerical results, the factor of 1.1 and 1.5 of the observations (FAC1.1 and FAC1.5) are applied for predictions of mean velocity and turbulent kinetic energy, respectively:

FAC1.1 $=\frac{1}{n} \sum_{i=1}^{n} N_{i}$ with $N_{i}=\left\{\begin{array}{ll}1 & \text { for } 0.91 \leq \frac{P_{i}}{O_{i}} \leq 1.1 \\ 0 & \text { else }\end{array}\right\}$

FAC1.5 $=\frac{1}{n} \sum_{i=1}^{n} N_{i}$ with $N_{i}=\left\{\begin{array}{ll}1 & \text { for } \quad 0.67 \leq \frac{P_{i}}{O_{i}} \leq 1.5 \\ 0 & \text { else }\end{array}\right\}$

with $P_{i}$ and $O_{i}$ the values obtained from CFD simulations and PIV experiments, respectively, and $n$ the number of data points. The values of FAC1.1 and FAC1.5 indicate the fraction of the considered data points, where the simulation results fall within a factor of 1.1 and 1.5 of the experimentally obtained values, respectively. As indicated by Schatzmann et al. (2010), FAC1.1 and FAC1.5 provide a clear picture of randomly occurring high or low differences between measured and predicted values of characteristic flow quantities. The predictions of the centerline mean velocity result in $\mathrm{FAC1} 1=0.91$, while the predictions of the centerline turbulent kinetic energy result in FAC1.5 $=0.64$. Compared with the results from 3D RANS simulations with the RNG $k$ - $\varepsilon$ turbulence model (see Khayrullina et al. 2019), the current 2D predicted values of the centerline mean velocity show a very similar agreement (i.e. FAC1.1 = 0.91 for $3 \mathrm{D}$ RANS), while the $2 \mathrm{D}$ values of the centerline turbulent kinetic energy show a worse agreement with the experimental data (i.e. FAC1.5 $=0.80$ for 3D RANS), which can - at least partly - be explained by the fact that turbulent kinetic energy is based on the two modeled normal Reynolds stress terms in the 2D simulation (instead of three terms as in the 3D simulation). The values of FAC1.1 and FAC1.5 obtained from the 2D simulations are considered to be within an acceptable range and allow a reduction of the computational demand for the large amount of simulations conducted in this study.

\section{Case studies}

A parametric study is performed for the case of an isothermal jet representing an air curtain that separates two environments subjected to a cross-jet pressure gradient, in which the influence of the momentum flux ratio, the jet height-to-width ratio and the jet discharge angle on the jet separation efficiency is evaluated. Similar to the validation study described in Section 2, this case study is performed with a water jet in a reduced-scale domain, representative of an air jet in a full-scale domain (geometric scaling factor $=1: 8)$.

\subsection{Computational geometry and grid}

Figure 6a shows the 2D computational geometry for the case study replicating to a large extent the computational geometry used in the validation study (Section 2 ). The jet widths at the nozzle exit $w_{\text {jet }}=8,12,16,20 \mathrm{~mm}$ (where $16 \mathrm{~mm}$ corresponds to the validation study) are studied resulting in height-to-width ratios of $\beta=45.0,30.0,22.5$ and 18.0, respectively. Additional jet discharge angles are considered in this case study: i.e. $\alpha_{0}=5^{\circ}, 10^{\circ}$, and $20^{\circ}$ (Figs. $6 \mathrm{c}-6 \mathrm{e}$, respectively). This resulted in 16 different computational geometries. Note that $w_{\text {jet }}$ does not change when varying the jet discharge angle. As in the validation study, the coarse grid is used based on a grid sensitivity analysis. 

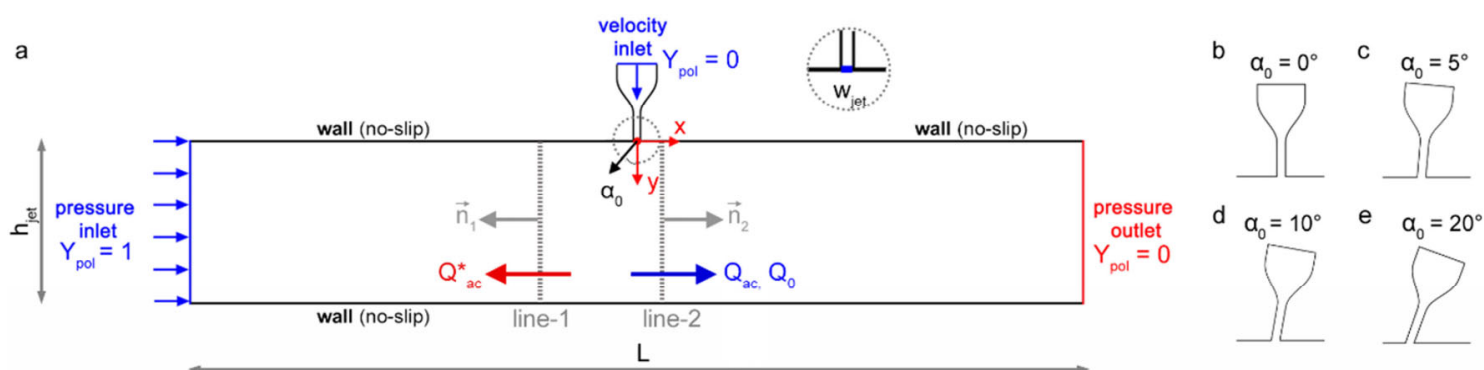

Fig. 6 (a) Computational geometry with indication of boundary conditions, (b-e) nozzle with: (b) $\alpha_{0}=0^{\circ}$; (c) $\alpha_{0}=5^{\circ}$; (d) $\alpha_{0}=10^{\circ}$; (e) $\alpha_{0}=$ $20^{\circ}$. The dashed lines on both sides of the jet indicate the lines used for the analysis of the results

\subsection{Boundary conditions}

In order to assess the AC separation efficiency, a cross-jet static pressure difference of $10 \mathrm{~Pa}$ is applied by imposing a $10 \mathrm{~Pa}$ static gauge pressure at the left side of the enclosure (along the height $h_{\mathrm{jet}}$ ) by means of a pressure inlet boundary condition and zero static gauge pressure at the right side of the enclosure by means of a pressure outlet boundary condition. The water in the computational domain is defined as a mixture of two species: clean water and a passive pollutant. The condition $Y_{\mathrm{pol}}+Y_{\mathrm{cl}}=1$, with $Y_{\mathrm{pol}}$ the pollutant mass fraction and $Y_{\mathrm{cl}}$ the clean water mass fraction, applies throughout the computational domain. The pollutant mass fraction imposed at the pressure inlet of the enclosure is $Y_{\text {pol }}=1$, while the pollutant mass fraction at the velocity inlet (nozzle inlet) and the pressure outlet on the right side of the enclosure is $Y_{\mathrm{pol}}=0$. The pollutant mass fraction is also specified at the pressure outlet as backflow can occur at this boundary. A range of mean velocities at the velocity inlet is considered, resulting in 416 cases with jet $\operatorname{Re}(=$ $\left(\left|V_{0}\right| w_{\text {jet }}\right) / v$, with $\left|V_{0}\right|$ the jet velocity magnitude at the nozzle exit) varying from 5,000 to 30,000 with a uniform step $(\Delta R e=1,000)$.

\subsection{Turbulence model and solver settings}

The settings are identical to those in the validation study (see Section 2.2.3). Convergence is considered to be achieved when the scaled residuals reach the following minimum values: $10^{-14}$ for $x$ and $y$ velocity, $10^{-13}$ for $k$ and $\varepsilon, 10^{-12}$ for continuity, and $10^{-8}$ for species transport.

\subsection{Evaluation parameters}

In this study the ratio of jet discharge momentum flux $\left(M_{\mathrm{jet}}\right)$ to cross-flow momentum flux $\left(M_{\mathrm{cf}}\right)$, called the momentum flux ratio $\gamma$, is used to characterize the jet:

$$
\gamma=\frac{M_{\text {jet }}}{M_{\mathrm{cf}}}=\frac{\rho_{\mathrm{w}} w_{\mathrm{jet}}\left|V_{0}\right|^{2}}{\rho_{\mathrm{w}} h_{\mathrm{jet}} U_{\mathrm{cf}}^{2}}=\frac{w_{\text {jet }}\left|V_{0}\right|^{2}}{h_{\mathrm{jet}} U_{\mathrm{cf}}^{2}}
$$

with $\left|V_{0}\right|$ the mean jet velocity magnitude at the nozzle exit $(y=0 \mathrm{~m})$ and $U_{\mathrm{cf}}$ the average mean velocity of the crossflow through the enclosure created by the pressure gradient for the case without an $\mathrm{AC}$ ( $U_{\mathrm{cf}}$ is equal to $\approx 0.13 \mathrm{~m} / \mathrm{s}$, which is representative of cross-flow velocity (air) in full scale equal to $0.25 \mathrm{~m} / \mathrm{s}$ ). Figure 7 shows the quadratic relationship between the jet Reynolds number and $\gamma$.

The jet separation efficiencies $\eta$ and $\eta^{*}$ are calculated according to Eq. (1) and Eq. (2), respectively. However, since there is no exfiltration through the opening in the case without an AC due to the imposed pressure gradient, Eq. (2) can, in this particular case, be simplified to:

$\eta^{*}=1-\frac{Q_{a c}+Q_{a c}^{*}}{Q_{0}}$

The transfer of the pollutant to the right side of the enclosure (infiltration) is computed along line- 2 at a distance $x_{2}=$ $0.05 \mathrm{~m}$ from the middle of the nozzle exit $(x=0 \mathrm{~m})$ (see Fig. 6a), with:

$Q_{i}=\int_{0}^{h_{\text {jet }}} Y_{\mathrm{pol}} \rho_{\mathrm{w}}\left(\vec{V} \cdot \overrightarrow{n_{2}}\right) \mathrm{d} y$

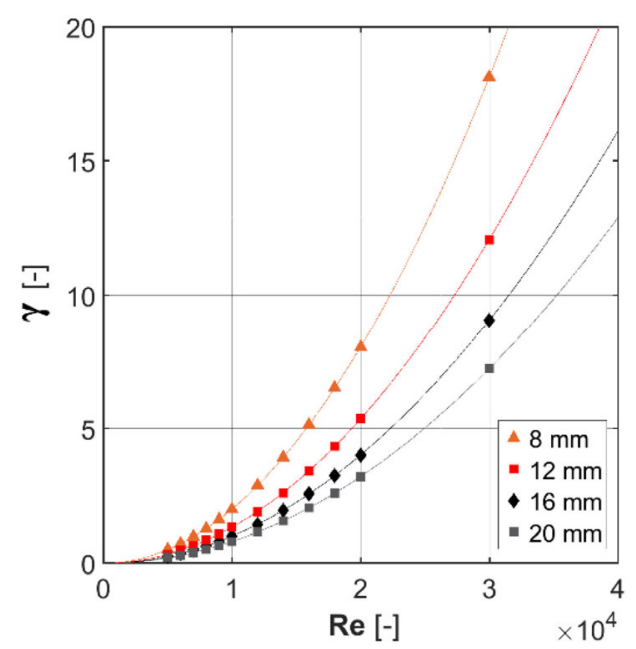

Fig. 7 Relation between jet Reynolds number and $\gamma=M_{\mathrm{jet}} / M_{\mathrm{cf}}$ for four jet widths 
with $Q_{i}$ the pollutant mass transfer rate from the left side to the right side (with or without air curtain, i.e. $Q_{\mathrm{ac}}$ or $Q_{0}$, respectively), $Y_{\text {pol }}$ the mass fraction of the pollutant, $\rho_{w}$ the water density, $\vec{V}$ the velocity vector, and $\vec{n}_{2}$ the outward vector normal to line-2, i.e. the vector corresponding to the direction of the pollutant mass transfer $Q_{i}$ as indicated in Fig. 6a. The transfer of clean water from the nozzle exit and from the right side of the enclosure to the left side (exfiltration) is computed along line- 1 at a distance $x_{1}=$ $-0.20 \mathrm{~m}$ from the middle of the nozzle exit $(x=0)$ (see Fig. 6a) with:

$Q_{\mathrm{ac}}^{*}=\int_{0}^{h_{\mathrm{jet}}} Y_{\mathrm{cl}} \rho_{\mathrm{w}}\left(\vec{V} \cdot \vec{n}_{1}\right) \mathrm{d} y$

with $Q_{\mathrm{ac}}^{*}$ the clean water mass transfer rate with air curtain to the left side of the enclosure, $Y_{\mathrm{cl}}$ the clean water mass fraction, and $\vec{n}_{1}$ the outward vector normal to the line-1, i.e. the vector corresponding to the direction of the clean water mass transfer $Q_{i}^{*}$ as indicated in Fig. 6a. The distances of $x_{1}=-0.20 \mathrm{~m}$ and $x_{2}=0.05 \mathrm{~m}$ are based on a sensitivity analysis with the aim to minimize the influence of jet flow on the results for species mass transfer.

\section{Results}

This section analyzes the influence of jet height-to-width ratio $(\beta)$, jet discharge angle $\left(\alpha_{0}\right)$ and jet momentum flux ratio $(\gamma)$ on jet separation efficiency. We define the "optimal momentum flux ratio" (optimal $\gamma$ ) as the ratio for which the modified separation efficiency $\eta^{*}$ (see Eq. (2)) reaches its highest value and we provide the values of the separation efficiencies $\eta$ (see Eq. (1)) that correspond to this optimal $\gamma$. In order to provide a more accurate estimation of the optimal $\gamma$ for different geometries, additional simulations have been performed for each computational geometry. These simulations are performed for a range of $\gamma$, for which the defined separation efficiencies $\eta^{*}$ reach the highest values, with a refined step of $\Delta R e=100$ for the jet.

4.1 Influence of jet height-to-width ratio on jet separation efficiency

Figure 8 provides the separation efficiencies $\eta$ and $\eta^{*}$ as a function of $\gamma$ for two different jet discharge angles: $\alpha_{0}=0^{\circ}$ and $20^{\circ}$, presented separately for each considered jet heightto-width ratio: $\beta=18$ (Figs. $8 \mathrm{a}, \mathrm{b}$ ), $\beta=22.5$ (Figs. $8 \mathrm{c}, \mathrm{d}$ ), $\beta=$ 30 (Figs. 8e,f) and $\beta=45$ (Figs. 8g,h). Each data point in the figure corresponds to one case with a certain momentum flux ratio of the jet and a certain jet discharge angle. The data for jets with discharge angles $\alpha_{0}=5^{\circ}$ and $10^{\circ}$ are excluded from the figure to ensure its readability.
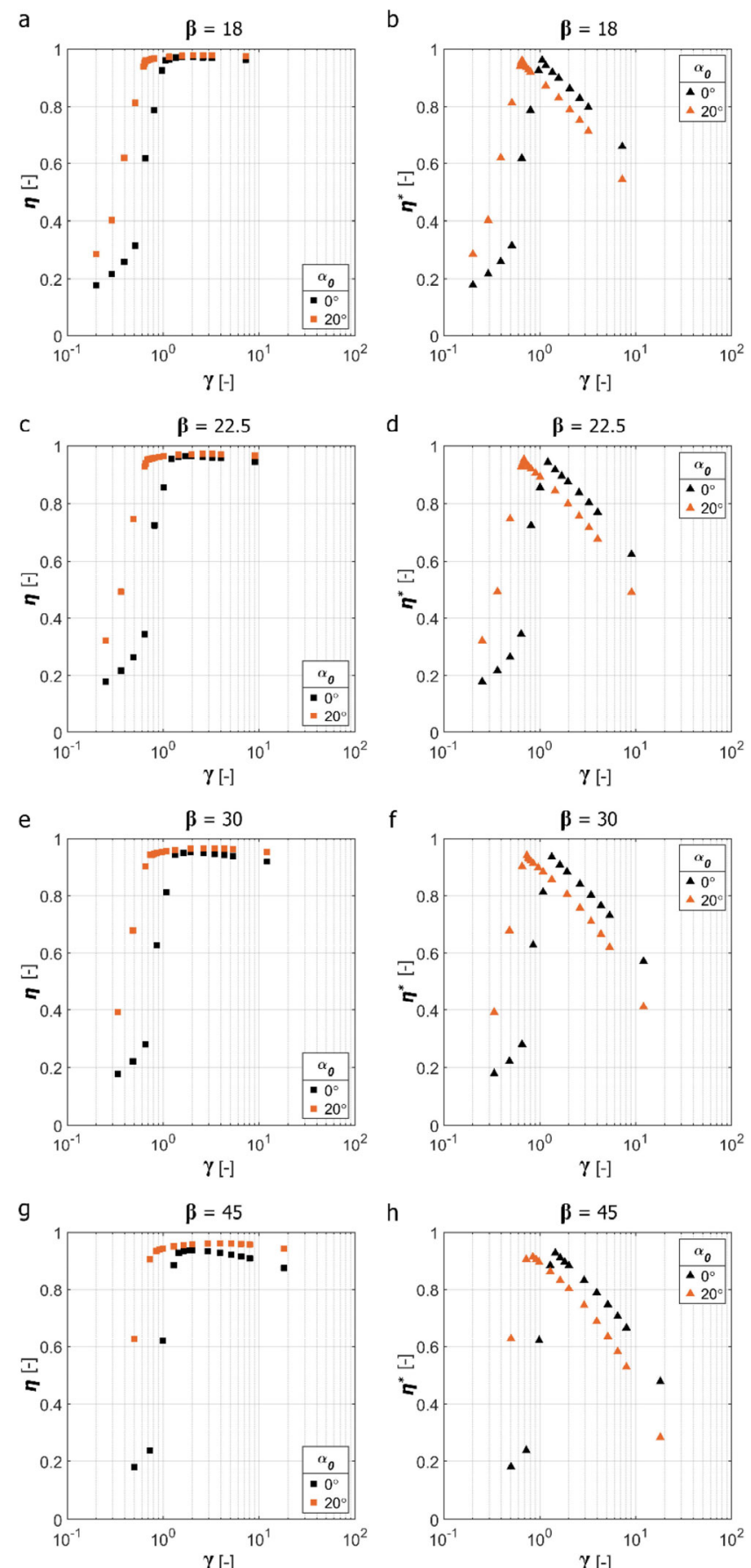

Fig. 8 Influence of jet height-to-width ratio $\beta$ on jet separation efficiencies $\eta$ and $\eta^{*}$ for jets with $\alpha_{0}=0^{\circ}$ and $20^{\circ}:(\mathrm{a}, \mathrm{b}) \beta=h_{\text {jet }} / w_{\text {jet }}=18$; (c,d) $\beta=22.5$; (e,f) $\beta=30$; (g,h) $\beta=45$

For jets with discharge angle $\alpha_{0}=0^{\circ}$, Figure 8 shows that starting from $\gamma=0.2$ until $\gamma \approx 1.5$ the separation efficiency $\eta$ (Figs. 8a,c,e,g) steeply increases from $18 \%$ to $97.2 \%$ for jets with $\beta=18$, to $96.4 \%$ for jets with $\beta=22.5$, to $95.3 \%$ for jets with $\beta=30$, and to $93.9 \%$ for jets with $\beta=45$. The modified separation efficiency $\eta^{*}$ (Figs. 8b,d,f,h) reaches 96.0\% for jets with $\beta=18,94.3 \%$ for jets with $\beta=22.5$, 93.7\% for jets with $\beta=30$, and $92.8 \%$ for jets with $\beta=45$. 
For $\gamma>1.5$ the separation efficiency $\eta$ gradually decreases to $96.2 \%, 94.5 \%, 92.0 \%$ and $87.6 \%$ for jets with $\beta=18,22.5$, 30 and 45 , respectively. The modified separation efficiency $\eta^{*}$ shows a steeper decrease to $66.1 \%, 62.4 \%, 57.1 \%$ and $47.9 \%$ for jets with $\beta=18,22.5,30$ and 45 , respectively, within the considered range of values of $\gamma$. Note that for each considered computational geometry the highest value of the modified separation efficiency $\eta^{*}$ is obtained at slightly different values of $\gamma$.

Similarly, for jets with discharge angle $\alpha_{0}=20^{\circ}$, the separation efficiency $\eta$ (Figs. 8a,c,e,g) steeply increases from $29 \%$ to $97.8 \%$ for jets with $\beta=18$, to $97.2 \%$ for jets with $\beta=22.5$, to $96.6 \%$ for jets with $\beta=30$, and to $96.2 \%$ for jets with $\beta=45$. The modified separation efficiency $\eta^{*}$ (Figs. 8b,d,f,h) reaches $95.9 \%$ for jets with $\beta=18,95.2 \%$ for jets with $\beta=22.5,94.2 \%$ for jets with $\beta=30$, and $91.5 \%$ for jets with $\beta=45$. After reaching the maximum value, the separation efficiency $\eta$ gradually decreases to $97.5 \%, 96.6 \%$, 95.4\% and $94.3 \%$ for jets with $\beta=18,22.5,30$ and 45 , respectively. The modified separation efficiency $\eta^{*}$ shows a decrease to $54.5 \%, 49.0 \%, 41.1 \%$ and $28.2 \%$ for jets with $\beta=$ $18,22.5,30$ and 45 , respectively, within the considered range of values of $\gamma$.

Table 1 provides the values of optimal $\gamma$ for each considered computational geometry $\left(\beta\right.$ and $\left.\alpha_{0}\right)$ and the corresponding values of the separation efficiencies $\eta$ and $\eta^{*}$. The results for jet discharge angle $\alpha_{0}=0^{\circ}$ show that the highest value of the modified separation efficiency $\eta^{*}=0.960$ and the corresponding value of the separation efficiency

Table 1 Optimal values of momentum flux ratio $\gamma=M_{\mathrm{jet}} / M_{\mathrm{cf}}$ and corresponding separation efficiencies $\eta$ and $\eta^{*}$ for the considered cases

\begin{tabular}{ccccc}
\hline$\beta[-]$ & $\alpha_{0}\left[^{\circ}\right]$ & $\gamma$, optimal $[-]$ & $\eta[-]$ & $\eta^{*}[-]$ \\
\hline \multirow{3}{*}{18.0} & 0 & 1.07 & 0.961 & 0.960 \\
& 5 & 0.93 & 0.963 & 0.963 \\
& 10 & 0.82 & 0.963 & 0.963 \\
& 20 & 0.66 & 0.961 & 0.959 \\
\hline \multirow{4}{*}{22.5} & 0 & 1.22 & 0.955 & 0.943 \\
& 5 & 1.01 & 0.958 & 0.956 \\
& 10 & 0.87 & 0.957 & 0.956 \\
30.0 & 20 & 0.69 & 0.953 & 0.952 \\
\hline \multirow{3}{*}{45.0} & 0 & 1.34 & 0.946 & 0.937 \\
& 5 & 1.10 & 0.948 & 0.947 \\
& 10 & 0.95 & 0.948 & 0.947 \\
& 20 & 0.74 & 0.945 & 0.942 \\
\hline \multirow{3}{*}{3} & 0 & 1.46 & 0.929 & 0.928 \\
& 5 & 1.21 & 0.937 & 0.936 \\
& 10 & 1.03 & 0.937 & 0.936 \\
& 20 & 0.85 & 0.936 & 0.915 \\
\hline
\end{tabular}

$\eta=0.961$ are obtained for a jet with $\beta=18$, i.e. for larger jet widths. In addition, for $\alpha_{0}=0^{\circ}$, the lowest modified separation efficiency $\eta^{*}=0.928$ and the corresponding value of the separation efficiency $\eta=0.929$ are obtained for jets with $\beta=45$ (i.e. the smallest jet width). Likewise, for jets with jet discharge angle $\alpha_{0}=20^{\circ}$ the highest values of the separation efficiency $\eta^{*}=0.959$ and corresponding $\eta=$ 0.961 are obtained for a jet with $\beta=18$, and the lowest value of the modified separation efficiency $\eta^{*}=0.915$ and corresponding $\eta=0.936$ are obtained for a jet with $\beta=45$. Similar findings are obtained for jet discharge angles $\alpha_{0}=5^{\circ}$ and $10^{\circ}$. For each considered jet discharge angle, the optimal $\gamma$ for jets with $\beta=18$ is lower than for jets with larger $\beta$ (i.e. smaller jet widths).

\subsection{Influence of jet discharge angle on jet separation efficiency}

Figure 9 provides the distributions of $\eta$ and $\eta^{*}$ as a function of $\gamma$ for two different jet height-to-width ratios: $\beta=18$ and $\beta=45$, which are presented separately for each considered jet discharge angle: $\alpha_{0}=0^{\circ}$ (Figs. 9a,b), $5^{\circ}$ (Figs. 9c,d), $10^{\circ}$ (Figs. 9e,f), and $20^{\circ}$ (Figs. 9g,h). Similar to the previous subsection, the data for jets with $\beta=22.5$ and 30 are excluded from the figure to ensure its readability.

The results for jets with $\beta=18$ show that the separation efficiency $\eta$ (Figs. 9a,c,e,g) reaches $97.2 \%$ for jets with $\alpha_{0}=0^{\circ}$, 97.1\% for jets with $\alpha_{0}=5^{\circ}, 97.3 \%$ for jets with $\alpha_{0}=10^{\circ}$, and $97.8 \%$ for jets with $\alpha_{0}=20^{\circ}$. The modified separation efficiency $\eta^{*}$ (Figs. 9b,d,f,h) reaches $96.0 \%$ for jets with $\alpha_{0}=$ $0^{\circ}, 96.3 \%$ for jets with $\alpha_{0}=5^{\circ}$ and $10^{\circ}$, and $95.9 \%$ for jets with $\alpha_{0}=20^{\circ}$.

With regard to jets with $\beta=45$, the separation efficiency $\eta$ (Figs. 9a,c,e,g) reaches $93.9 \%$ for jets with $\alpha_{0}=0^{\circ}, 94.1 \%$ for jets with $\alpha_{0}=5^{\circ}, 94.4 \%$ for jets with $\alpha_{0}=10^{\circ}$, and $96.2 \%$ for jets with $\alpha_{0}=20^{\circ}$. The modified separation efficiency $\eta^{*}$ (Figs. 9b,d,f,h) reaches $92.8 \%$ for jets with $\alpha_{0}=0^{\circ}, 93.6 \%$ for jets with $\alpha_{0}=5^{\circ}$ and $10^{\circ}$, and $91.5 \%$ for jets with $\alpha_{0}=20^{\circ}$.

The results in Table 1 show that for jets with $\beta=18$ the highest value of the modified separation efficiency $\eta^{*}=0.963$ and the corresponding value of the separation efficiency $\eta=0.963$ are obtained for jets with $\alpha_{0}=5^{\circ}$ and $10^{\circ}$. Likewise, for jets with $\beta=45$ the highest value of $\eta^{*}=0.936$ and corresponding value of $\eta=0.937$ are obtained for jets with $\alpha_{0}=5^{\circ}$ and $10^{\circ}$. Similar findings are obtained for jets with $\beta=22.5$ and 30 . Overall, inclined jets with $\alpha_{0}=5^{\circ}$ and $10^{\circ}$ provide slightly higher separation efficiencies than straight $\left(\alpha_{0}=0^{\circ}\right)$ jets and jets with $\alpha_{0}=20^{\circ}$. Note that optimal $\gamma$ for jets with $\alpha_{0}=20^{\circ}$ are lower than for jets with smaller discharge angles. The optimal $\gamma$ for straight jets $\left(\alpha_{0}=0^{\circ}\right)$ are highest compared to those of jets with discharge angles $\alpha_{0}=5^{\circ}, 10^{\circ}, 20^{\circ}$. 
a
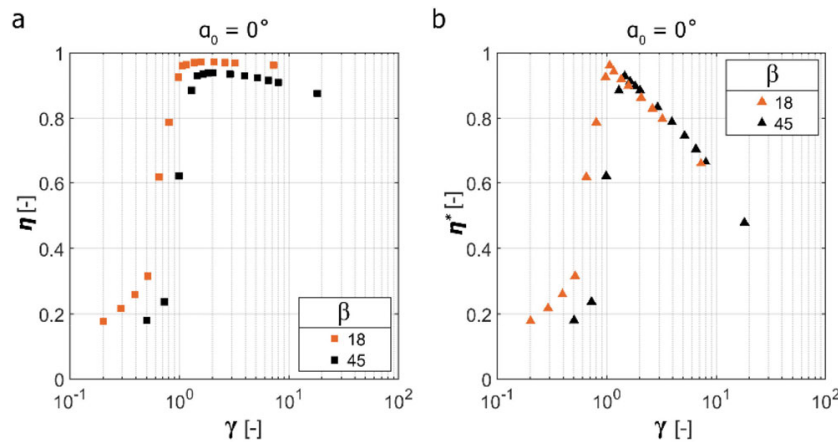

C
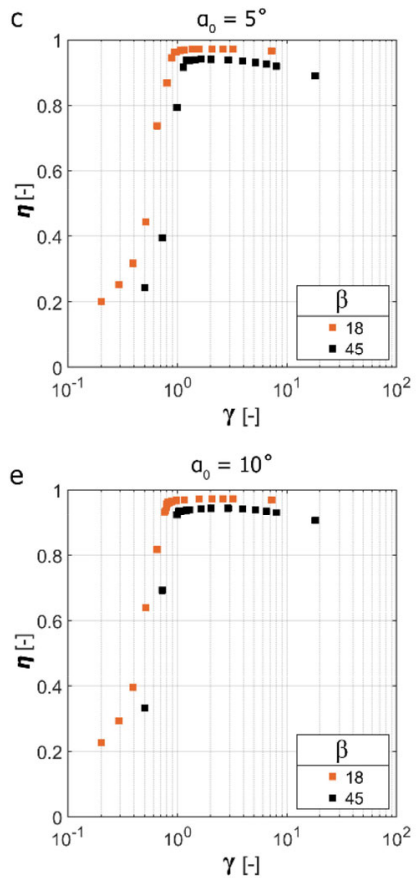

9

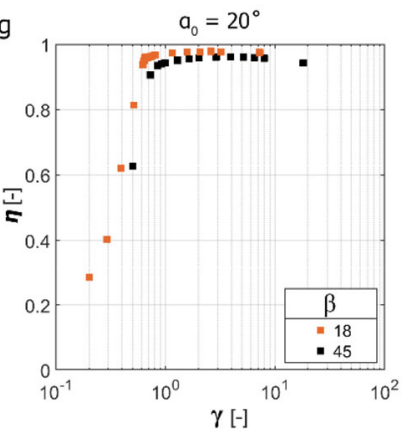

d

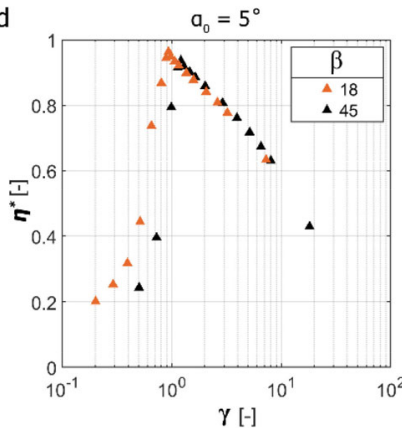

f

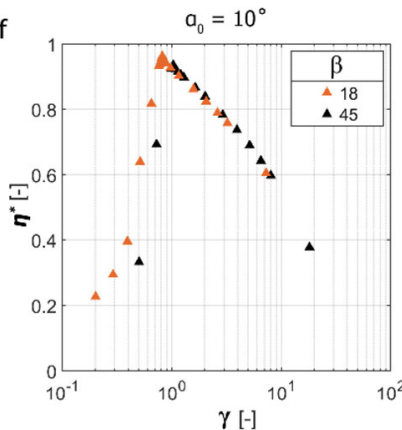

h

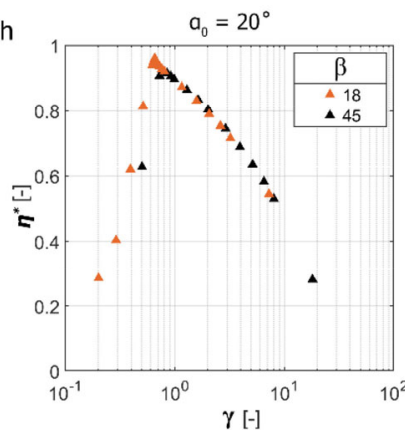

Fig. 9 Influence of jet discharge angle $\alpha_{0}$ on separation efficiencies $\eta$ and $\eta^{*}$ for jets with $\beta=18$ and 45: (a,b) $\alpha_{0}=0^{\circ}$; (c,d) $\alpha_{0}=5^{\circ}$; (e,f) $\alpha_{0}=10^{\circ} ;(\mathrm{g}, \mathrm{h}) \alpha_{0}=20^{\circ}$

4.3 Influence of momentum flux ratio on jet separation efficiency

Figure 10 shows the distributions of $\eta$ and $\eta^{*}$ as a function of $\gamma$. Each data point in the figure corresponds to one case with a jet at a certain $R e, \beta$ and $\alpha_{0}$. For each case two data points are shown that present $\eta$ and $\eta^{*}$, respectively. The shape of the distribution of $\eta$ resembles those provided by

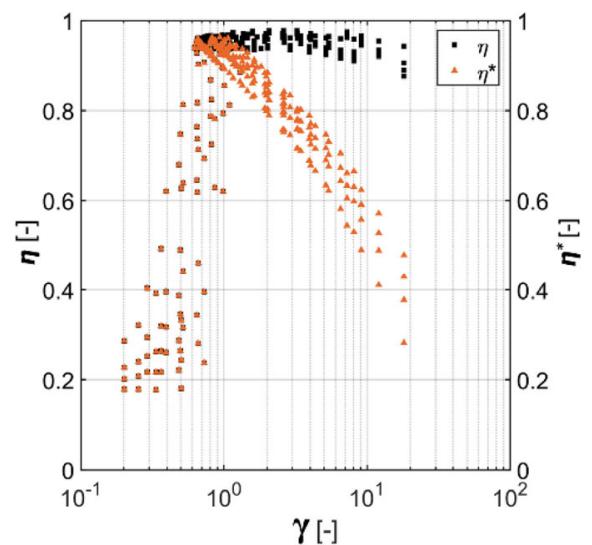

Fig. 10 Separation efficiencies $\eta(\bullet)$ and $\eta^{*}(\Delta)$ as a function of momentum flux ratio $\gamma$

e.g. Costa et al. (2006), Foster et al. (2006) and Frank and Linden (2015). Figures 11a-11c show the distributions of dimensionless mean velocity magnitude $\left(|V| /\left|V_{0}\right|\right)$ for the jet with $\beta=22.5, \alpha_{0}=0^{\circ}$ and for three $\gamma=0.25,1.22$ and 9.07, respectively.

For $\gamma=0.25$, the jet momentum flux cannot compensate the cross-jet pressure gradient and does not reach the floor, i.e. so-called breakthrough of the jet occurs (Fig. 11a). The separation efficiencies here are $\eta=\eta^{*}=0.178$. From $\gamma=$ 0.25 to $\gamma=1.22$ the separation efficiencies $\eta$ and $\eta^{*}$ are steeply increasing from 0.18 to 0.955 and 0.943 , respectively. At $\gamma=1.22$, the jet reaches the ground providing an aerodynamic sealing (Fig. 11b). The separation efficiency $\eta^{*}$ starts to decrease for $\gamma>1.22$, while the separation efficiency $\eta$ starts to decrease for $\gamma>1.97$, with a steeper decrease for $\eta^{*}$ compared to that for $\eta$. At $\gamma=9.07$ (Fig. 11c) the jet provides an aerodynamic sealing with $\eta=0.945$ and $\eta^{*}=$

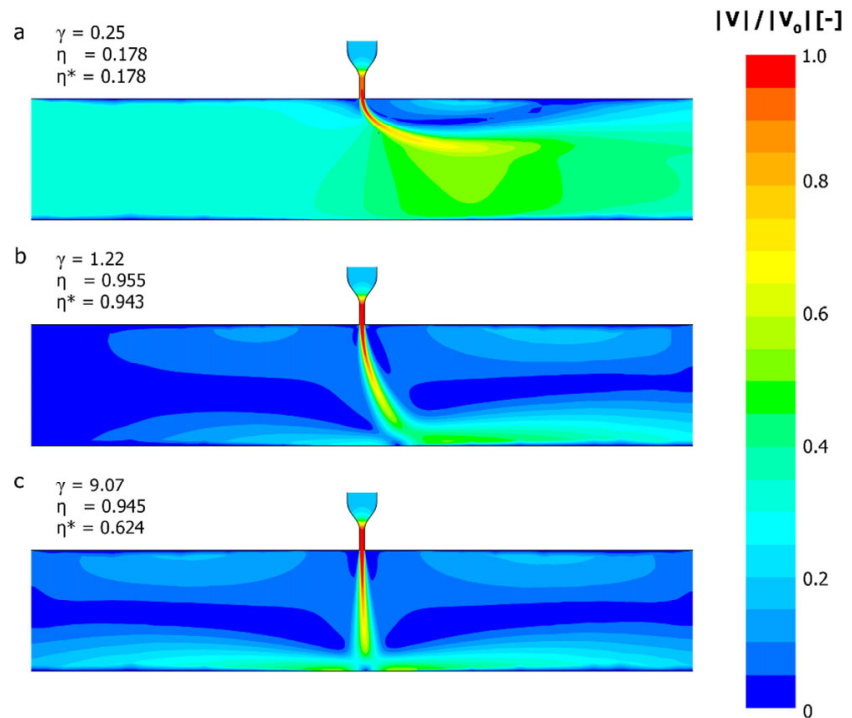

Fig. 11 Contours of dimensionless mean velocity magnitude ( $\left.|V| /\left|V_{0}\right|\right)$ for $\beta=22.5$ for three momentum flux ratios: (a) $\gamma=0.25$; (b) $\gamma=1.22$; (c) $\gamma=9.07$ 
0.624. The explanation for this steep decrease of the separation efficiency $\eta^{*}$ is that at $\gamma>1.22$ a significant part of the supplied water from the nozzle exit flows to the left side of the enclosure, resulting in a lower modified separation efficiency. Moreover, higher momentum flux ratios cause more entrainment of the ambient fluid into the jet. In the considered case the pollutant mass transfer from the left part of the enclosure into the right part was $18 \%$ lower for $\gamma=1.22$ $\left(Q_{\mathrm{ac}}=2.26 \mathrm{~kg} / \mathrm{s}\right)$ than that for $\gamma=9.07\left(Q_{\mathrm{ac}}=2.76 \mathrm{~kg} / \mathrm{s}\right)$.

The results in Table 1 show that:

- The optimal $\gamma$ ranges from 0.66 to 1.46 . The highest value ( $\gamma=1.46)$ occurs for a jet with $\beta=45$ at $\alpha_{0}=0^{\circ}$. The lowest value $(\gamma=0.66)$ occurs for a jet with $\beta=18$ at $\alpha_{0}=20^{\circ}$.

- The modified separation efficiencies $\eta^{*}$, i.e. the values corresponding to the optimal $\gamma$ in Table 1, range from 0.915 to 0.963 . The highest value $\left(\eta^{*}=0.963\right)$ occurs for $\beta=18$ at $\alpha_{0}=5^{\circ}$ and $10^{\circ}$. The lowest value $\left(\eta^{*}=0.915\right)$ occurs for $\beta=45$ at $\alpha_{0}=20^{\circ}$.

- The separation efficiency $\eta$ corresponding to the optimal $\gamma$ ranges from 0.929 to 0.963 . The highest value $(\eta=$ $0.963)$ occurs for $\beta=18$ at $\alpha_{0}=5^{\circ}$ and $10^{\circ}$. The lowest value $(\eta=0.929)$ occurs for $\beta=45$ at $\alpha_{0}=0^{\circ}$.

The value of the optimal $\gamma$ for a jet with $\beta=18$ at $\alpha_{0}=$ $20^{\circ}$ is lower than for jets with other $\beta$ at the same discharge angle $\alpha_{0}=20^{\circ}$. Figure 12 provides the distributions of pollutant mass fractions for jets with $\gamma=0.66: \beta=18$ at $\alpha_{0}=$ $0^{\circ}$ and $\alpha_{0}=20^{\circ}$ and $\beta=45$ at $\alpha_{0}=20^{\circ}$. Figure 12 shows that a significant amount of pollutant is transferred through the jet to the right side of the enclosure for the cases with jet $\beta=18$ at $\alpha_{0}=0^{\circ}\left(Q_{\mathrm{ac}}=19.13 \mathrm{~kg} / \mathrm{s}, \eta=0.618\right)$ and $\beta=45$ at $\alpha_{0}=20^{\circ}\left(Q_{\mathrm{ac}}=9.61 \mathrm{~kg} / \mathrm{s}, \eta=0.808\right)$ compared to the case with $\beta=18$ at $\alpha_{0}=20^{\circ}\left(Q_{\mathrm{ac}}=2.06 \mathrm{~kg} / \mathrm{s}, \eta=0.961\right)$. Therefore, the combination of two factors, such as smaller jet heightto-width ratio and larger jet discharge angle, helps a jet to counteract the cross-jet pressure gradient. Figure $12 \mathrm{a}$ also shows that, for the case with $\beta=18$ at $\alpha_{0}=20^{\circ}$, the pollutant concentration on the left side of the enclosure remains much lower compared to the cases with $\beta=18$ at $\alpha_{0}=0^{\circ}$ and $\beta=45$ at $\alpha_{0}=20^{\circ}$, where $Y_{\text {pol }} \approx 1$. Thus, mixing occurs between "polluted" fluid in the left part of the enclosure with the fluid from the nozzle exit and the ambient fluid from the right part of the enclosure. For the case with $\beta=18$ and $\alpha_{0}=20^{\circ}$, the transfer of clean water from the nozzle exit and from the right side of the enclosure to the left side $Q_{\mathrm{ac}}^{*}=0.025 \mathrm{~kg} / \mathrm{s}$, while for the cases with $\beta=18$ and $\alpha_{0}=0^{\circ}$, and $\beta=45$ and $\alpha_{0}=20^{\circ}$ the values of $Q_{\mathrm{ac}}^{*}$ are equal to 0 . In combination with the above mentioned values of pollutant mass transfer $Q_{\mathrm{ac}}$ this results in the higher value of the modified separation efficiency $\left(\eta^{*}=\right.$ 0.959) for the case with $\beta=18$ and $\alpha_{0}=20^{\circ}$ than for the cases with $\beta=18$ and $\alpha_{0}=0^{\circ}\left(\eta^{*}=0.618\right)$, and $\beta=45$ and $\alpha_{0}=20^{\circ}\left(\eta^{*}=0.808\right)$.

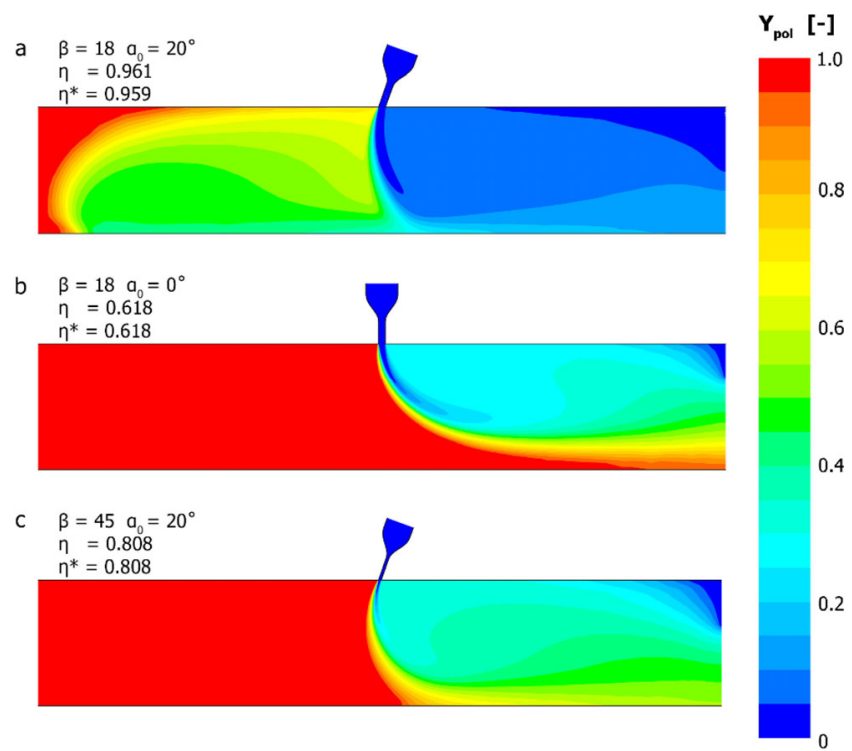

Fig. 12 Distributions of pollutant mass fraction $Y_{\text {pol }}$ for the cases with $\gamma=0.66$ : (a) $\beta=18$ at $\alpha_{0}=20^{\circ}$; (b) $\beta=18$ at $\alpha_{0}=0^{\circ}$; (c) $\beta=45$ at $\alpha_{0}=20^{\circ}$

\subsection{Deflection modulus}

The deflection modulus can also be defined as the dimensionless ratio of the magnitude of the discharge momentum flux of the jet to the transverse forces acting on the jet due to a cross-jet pressure gradient across the doorway (e.g. Howell and Shibata 1980, Sirén 2003; Frank and Linden 2015):

$D_{\mathrm{m}}=\frac{\rho w_{\text {jet }}\left|V_{0}\right|^{2}}{\Delta P h_{\text {jet }}}$

with $\Delta P$ the static pressure difference over the jet. In this section, the focus is on the minimum deflection modulus $D_{\mathrm{m}, \min }$ that is reached at the conditions where jet breakthrough just does not occur yet. For this, the approach also applied by Sirén (2003) is adopted that is based on the momentum balance. Assuming that the flow is steady-state and neglecting gravity and viscous forces, the force associated with the change in jet momentum flux in the $x$-direction (i.e. horizontal direction) is equal to the force associated with the cross-jet pressure gradients in $x$-direction (Fig. 13):

$\int_{0}^{h_{\text {jet }}} \rho U(\vec{V} \cdot \vec{n}) \mathrm{d} y=\Delta P h_{\text {jet }}=\left(P_{1}-P_{\mathrm{r}}\right) h_{\text {jet }}$

The left-hand side of Eq. (12) can be written as:

$\int_{0}^{h_{\text {jet }}} \rho U(\vec{V} \cdot \vec{n}) \mathrm{d} y=\rho w_{\text {jet }}\left|V_{0}\right|^{2} \sin \alpha_{0}-\rho w_{\text {jet }}^{\prime}|V|^{2} \sin \alpha$

with $U$ the mean lateral ( $x$-direction) velocity component, 


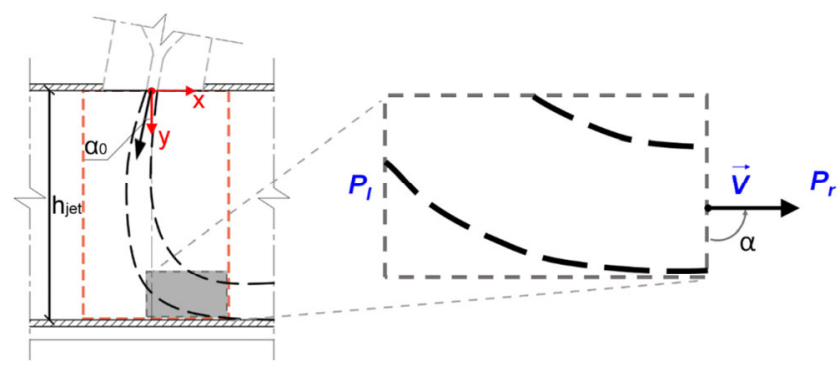

Fig. 13 Schematic of the jet used for deriving the minimum deflection modulus. The orange dashed rectangle indicates the contour for the momentum balance

$w_{\text {jet }}^{\prime}$ and $\alpha$ the width and the angle of the jet at the impingement region near the floor. Equation (13) is the $x$-component of the momentum balance applied to the contour indicated by the orange dashed rectangle in Fig. 13 . In addition, it is assumed that the jet momentum flux along the jet centerline is conserved (Rajaratnam 1976; Frank and Linden 2015):

$\rho w_{\text {jet }}\left|V_{0}\right|^{2}=\rho w_{\text {jet }}^{\prime}|V|^{2}$

The value of $D_{\mathrm{m} \text {,min }}$ is found when jet breakthrough occurs at $y=h_{\text {jet }}$ assuming that the jet angle $\alpha=-90^{\circ}$, i.e. when the jet is bent to the right side of the enclosure. Combining Eqs. (11)-(14) yields:

$$
D_{\mathrm{m}, \min }=\frac{1}{1+\sin \alpha_{0}}
$$

Table 2 lists the values of $D_{\mathrm{m}, \min }$ calculated based on the simplified Eq. (15) for the four jet discharge angles $\left(\alpha_{0}=0^{\circ}\right.$, $\left.5^{\circ}, 10^{\circ}, 20^{\circ}\right)$ and the corresponding values of $\gamma_{\min }=$ $M_{\mathrm{jet}, \text { min }} / M_{\mathrm{cf}}$. In addition, the values of $\gamma_{\mathrm{sf}}=M_{\mathrm{jet}, \mathrm{sf}} / M_{\mathrm{cf}}$ are provided, which are the values of $\gamma_{\min }$ corrected by a safety factor of 2, as recommended in previous studies (e.g. Hayes and Stoecker 1969a, Foster et al. 2006; Van Belleghem et al. 2012). Moreover, a comparison is provided between these values of $\gamma_{\min }$ and the values of the optimal momentum flux ratios $\gamma_{\mathrm{CFD}}=M_{\mathrm{jet}, \mathrm{CFD}} / M_{\mathrm{cf}}$ as obtained from the CFD simulations (see Table 1 with the values of $\gamma$ for four jet height-to-width ratios and for four jet discharge angles). The relative difference between these two quantities is calculated as:

$$
\delta \gamma=\frac{\gamma_{\mathrm{CFD}}-\gamma_{\mathrm{sf}}}{\gamma_{\mathrm{CFD}}}
$$

Figure 14 shows the values of the optimal $\gamma$ for four different $\alpha_{0}$ and four different $\beta$ together with distribution of $\gamma_{\mathrm{sf}}$.

The results in Table 2 show that the highest minimum value of the momentum flux ratio required to prevent a breakthrough of the jet and defined analytically in combination with a safety factor of $2\left(\gamma_{\mathrm{sf}}=1.16\right)$ is obtained for jets with $\alpha_{0}=0^{\circ}$. The lowest minimum value of $\gamma_{\mathrm{sf}}=$ 0.87 is obtained for a jet with $\alpha_{0}=20^{\circ}$. Note that the analytical method to define the minimum required momentum flux ratio considered in this study does not depend on the jet height-to-width ratio $\beta$, but solely on the jet discharge angle $\alpha_{0}$. In contrast, the values of the optimal $\gamma_{\mathrm{CFD}}$ depend on both $\alpha_{0}$ and $\beta$. The values of $\gamma_{\mathrm{CFD}}$ range from 1.07 to 1.46 for jets with $\alpha_{0}=0^{\circ}$, from 0.93 to 1.21 for jets with $\alpha_{0}=5^{\circ}$, from 0.82 to 1.03 for jets with $\alpha_{0}=10^{\circ}$, and from 0.66 to 0.85 for jets with $\alpha_{0}=20^{\circ}$. The highest value of $\gamma_{\text {CFD }}=1.46$ occurs for a jet with $\beta=45$ and $\alpha_{0}=0^{\circ}$ and the lowest value $\left(\gamma_{\mathrm{CFD}}=0.66\right)$ occurs for a jet with $\beta=$ 18 and $\alpha_{0}=20^{\circ}$. The results also show that the difference between the momentum flux ratios $\gamma_{\mathrm{sf}}$ obtained by Eq. (15) and corrected with a safety factor of 2 , and the values of optimal $\gamma_{\text {CFD }}$ reaches $\delta \gamma=-31.2 \%$ for the jet with $\beta=18$ and $\alpha_{0}=20^{\circ}$. These deviations are related to the complex flow dynamics of the jet in a cross-flow and to the assumptions made in defining the minimum jet deflection modulus (Eq. 15). As mentioned before, Eq. (15) does not take into account the jet height-to-width ratio. It also does not take into account the mixing of the jet with the ambient fluid, while optimal momentum flux ratios $\gamma_{\text {CFD }}$ defined with CFD simulations consider the potential transfer of the jet flow to the left part of the enclosure and clean water transfer from the right to the left part of the enclosure.

Figure 14 illustrates the common trend that both $\gamma_{\mathrm{sf}}$ and $\gamma_{\text {CFD }}$ decrease with increasing jet discharge angle $\alpha_{0}$.

Table 2 Values of deflection modulus $\mathrm{D}_{\mathrm{m} \text {,min }}$ and corresponding momentum flux ratios as determined by Eq. (15) in combination with a

\begin{tabular}{|c|c|c|c|c|c|c|c|c|c|c|c|}
\hline \multirow[b]{3}{*}{$\alpha_{0}\left[^{\circ}\right]$} & \multirow{3}{*}{$\begin{array}{c}D_{\mathrm{m}, \min }[-] \\
\text { (Eq. 15) }\end{array}$} & \multirow[b]{3}{*}{$\gamma_{\min }[-]$} & \multirow[b]{3}{*}{$\gamma_{\mathrm{sf}}[-]$} & \multicolumn{4}{|c|}{$\gamma_{\text {CFD }}[-]$} & \multicolumn{4}{|c|}{$\delta \gamma[\%]$} \\
\hline & & & & \multicolumn{4}{|c|}{$\beta$} & \multicolumn{4}{|c|}{$\beta$} \\
\hline & & & & 18 & 22.5 & 30 & 45 & 18 & 22.5 & 30 & 45 \\
\hline 0 & 1.00 & 0.58 & 1.16 & 1.07 & 1.22 & 1.34 & 1.46 & $-8.6 \%$ & $4.7 \%$ & $13.3 \%$ & $20.4 \%$ \\
\hline 5 & 0.92 & 0.53 & 1.07 & 0.93 & 1.01 & 1.10 & 1.21 & $-15.0 \%$ & $-5.9 \%$ & $2.8 \%$ & $11.6 \%$ \\
\hline 10 & 0.85 & 0.50 & 0.99 & 0.82 & 0.87 & 0.95 & 1.03 & $-20.8 \%$ & $-13.8 \%$ & $-4.3 \%$ & $3.8 \%$ \\
\hline 20 & 0.75 & 0.43 & 0.87 & 0.66 & 0.69 & 0.74 & 0.85 & $-31.2 \%$ & $-25.5 \%$ & $-17.1 \%$ & $-1.9 \%$ \\
\hline
\end{tabular}
safety factor and by CFD, and deviations between the latter two parameters. 


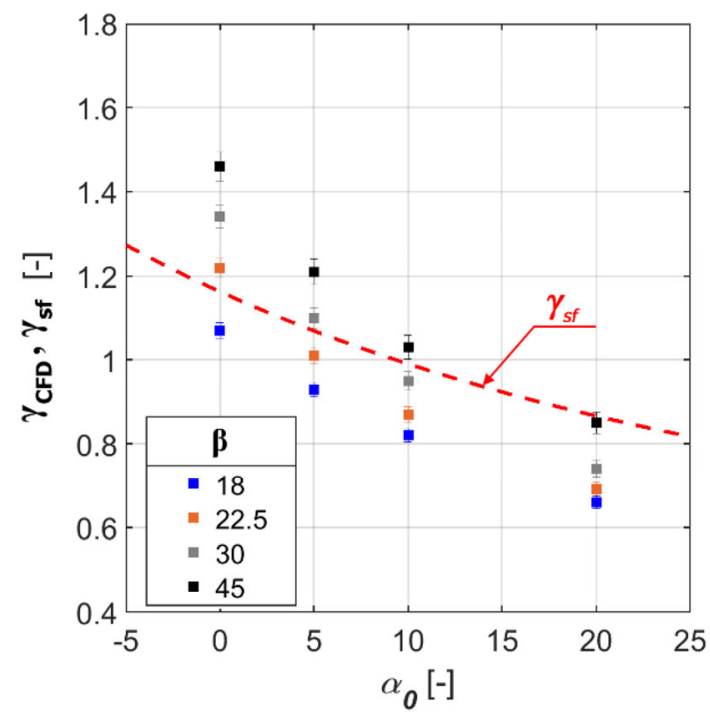

Fig. 14 Optimal values of momentum flux ratios obtained from CFD simulations. Error bars indicate the possible deviation of values of momentum flux ratios due to the applied step of $\Delta R e=$ 100. Red dashed line represents minimum required momentum ratios obtained based on Eq. (15) in combination with a safety factor $\left(\gamma_{\mathrm{sf}}\right)$

\section{Discussion}

This paper presents the results of a numerical study of a PTIJ (representing an air curtain) at Reynolds numbers ranging from 5,000 to 30,000. The influence of several jet parameters, i.e. the momentum flux ratio, the jet heightto-width ratio, and the jet discharge angle, on the jet separation efficiency is evaluated with $2 \mathrm{D}$ steady RANS CFD simulations. Based on this analysis optimal values of the momentum flux ratio are defined when the jet reaches its maximum modified separation efficiency. This modified separation efficiency does not only take infiltration into account but also exfiltration. Finally, the minimum deflection modulus required to prevent breakthrough of the jet and the corresponding minimum momentum flux ratios are defined based on a simple analytical equation and by applying a safety factor according to Hayes and Stoecker (1969a). These minimum momentum flux ratios are compared to the values of the optimal momentum flux ratios obtained from the CFD simulations.

\subsection{Applicability of steady RANS CFD simulations}

Although large eddy simulations (LES) are intrinsically superior to steady RANS simulations, a review of the literature on CFD in building simulation for outdoor and indoor applications shows that the latter are frequently used in both science and engineering and in both basic and applied research (Blocken 2018). Although future research will include LES as well, the validation study in the present paper has indicated that steady RANS was a suitable choice.

5.2 Applicability of the analytical equation to define the minimum deflection modulus

The analytical equation to determine the minimum deflection modulus is based on the principle of momentum conservation. It might be used in the initial estimation of the AC settings to prevent breakthrough of the jet. The analytical equation is simple and very easy to use but it has the following limitations:

- Mixing processes between the jet and the ambient environment are not taken into account.

- The specific influence of the jet height-to-width ratio is not taken into account.

- The assumption that jet breakthrough occurs at $y=h_{\text {jet }}$ when the jet angle $\alpha=-90^{\circ}$ does not take into account realistic jet behavior.

- The equation will be less reliable in more complex situations, e.g. involving pollutant mass transfer at densities different than density of the ambient fluid or with localized sources.

\subsection{Future work}

Based on this study the following recommendations for future work can be made:

- Future studies can consider angles larger than $\alpha_{0}=20^{\circ}$ and investigate their influence on the jet separation efficiencies and the corresponding optimal momentum flux ratios. Moreover, the optimum jet discharge angle can be defined to obtain the highest separation efficiency.

- This study considered a given constant cross-jet pressure that was uniform along the height of the opening. Future studies should consider a range of pressure gradients and their influence on the optimal momentum flux ratios. In real situations, pronounced pressure gradients can be present along the jet height and the jet can be imposed to highly dynamic cross-jet wind pressure (i.e. when ACs separate outdoor and indoor environment). Such more case-specific situations should be taken into account for actual/real case studies with AC implementation.

- This study considered an isothermal situation. Real situations often involve differences in air temperature between the indoor and outdoor environments, which also induces cross-jet pressure differences. Moreover, the presence of natural or mechanical ventilation in the building has its influence on the flow inside the building.

- The optimal momentum flux ratios were determined with CFD simulations for a steady-state situation. Future studies can consider transient environmental conditions such as moving public, sliding doors and changing wind 
speeds, which can affect the actual separation efficiency of the AC. Such effects could be included by means of safety factors that can be determined by transient CFD simulations or experiments.

- In the present paper, the nozzle aspect ratio was $A R=$ 18.75 and the jet height-to-width ratio was $\beta=22.5$. However, it should be noted that in real cases, the width of an air curtain may not be large enough to be considered as a $2 \mathrm{D}$ case. It is expected that the edge effects would cause a decrease in separation efficiency. Further research should address the separation efficiency and modified separation efficiency of such air curtain systems.

- In this study, the CFD simulations included the geometry of the nozzle, similar to the experimental setup. However, given the large nozzle aspect ratio $(A R=18.75)$ and the observation that the mean velocity and turbulence intensity profiles at the nozzle exit were quite uniform, an alternative option can be to exclude the nozzle. This would allow a reduction of the computational cost. For lower nozzle aspect ratios and for different types of nozzles, further research should investigate to what extent the profiles of mean velocity and turbulence intensity at the nozzle exit can be approximated by uniform profiles. If uniform profiles are applicable, the nozzle geometry can be excluded from the CFD simulations. If not, at least a separate CFD simulation should be made to extract the proper profiles of mean velocity and turbulence intensity at the nozzle exit.

\section{Conclusions}

The generic situation of an isothermal PTIJ (air curtain) at $5,000<R e<30,000$ separating two environments subjected to a cross-jet pressure gradient is studied using $2 \mathrm{D}$ steady RANS CFD simulations. The study involves a parametric analysis of the influence of several jet parameters (momentum flux ratio, jet height-to-width ratio, jet discharge angle) on the jet separation efficiency. Four jet height-to-width ratios $(\beta=18,22.5,30,45)$ and four jet discharge angles $\left(\alpha_{0}=0^{\circ}\right.$, $\left.5^{\circ}, 10^{\circ}, 20^{\circ}\right)$ are considered. For each jet height-to-width ratio and jet discharge angle, the optimal ratio of jet discharge momentum flux to jet cross-flow momentum flux (optimal momentum flux ratio) at which the modified separation efficiency of an AC reaches its maximum value is defined. At momentum flux ratios higher than the optimal value the jet hits the floor at higher velocities, resulting in excessive mixing between the jet and the ambient environment. In practice that would result in energy dissipation from the jet to the outdoor environment. Therefore, a too high momentum flux ratio causes an increased energy consumption by the air-curtain fan which should be prevented. Finally, the minimum deflection modulus to prevent breakthrough of the jet and its corresponding minimum momentum flux ratios are obtained based on a simplified equation and are compared to the values of optimal momentum flux ratios obtained from the CFD simulations. The following conclusions are provided:

- The highest modified separation efficiency, i.e. $\eta^{*}=0.963$, is obtained for a jet with $\beta=18$ (at $\alpha_{0}=5^{\circ}$ and $10^{\circ}$ ).

- The lowest modified separation efficiency $\eta^{*}=0.915$ is obtained for a jet with $\beta=45$ at $\alpha_{0}=20^{\circ}$.

- The highest value of the optimal momentum flux ratio $\gamma=1.46$ occurs for a jet with $\beta=45$ at $\alpha_{0}=0^{\circ}$.

- The lowest value of the optimal momentum flux ratio $\gamma=0.66$ occurs for a jet with $\beta=18$ and $\alpha_{0}=20^{\circ}$.

- Jets with smaller height-to-width ratios provide a higher modified separation efficiency than jets with larger height-to-width ratios. Moreover, jets with the smallest height-to-width ratio $(\beta=18)$ reach their maximum modified separation efficiency at lower optimal momentum flux ratios.

- Inclined jets with discharge angles $\alpha_{0}=5^{\circ}$ and $10^{\circ}$ provide a higher modified separation efficiency $\eta^{*}$ than straight $\left(\alpha_{0}=0^{\circ}\right)$ jets and jets with discharge angle $\alpha_{0}=20^{\circ}$.

- The values of the optimal momentum flux ratio calculated based on the CFD simulations range from $1.07<\gamma<1.46$ for jets at discharge angle $\alpha_{0}=0^{\circ}, 0.93<\gamma<1.21$ for jets at discharge angle $\alpha_{0}=5^{\circ}, 0.82<\gamma<1.03$ for jets at discharge angle $\alpha_{0}=10^{\circ}$, and $0.66<\gamma<0.85$ for jets at discharge angle $\alpha_{0}=20^{\circ}$. The highest values of $\gamma$ for each jet discharge angle correspond to the largest jet heightto-width ratio $(\beta=45)$.

- The values of the optimal momentum flux ratio determined based on the CFD simulations deviate by up to $31.2 \%$ from the values defined by the simple analytical equation including a safety factor. This simplified equation can be used for the initial estimation of the required AC conditions to provide aerodynamic sealing in a given situation. However, in order to provide more accurate information, one should resort to the methods that better take into account the jet dynamics, e.g. CFD simulations with RANS modeling, or more advanced modeling with LES.

\section{Acknowledgements}

Twan van Hooff is currently a postdoctoral fellow of the Research Foundation - Flanders (FWO) and acknowledges its financial support (project FWO 12R9718N). The authors acknowledge the partnership with ANSYS CFD.

Open Access: This article is licensed under a Creative Commons Attribution 4.0 International License, which permits use, sharing, adaptation, distribution and repro- 
duction in any medium or format, as long as you give appropriate credit to the original author(s) and the source, provide a link to the Creative Commons licence, and indicate if changes were made.

The images or other third party material in this article are included in the article's Creative Commons licence, unless indicated otherwise in a credit line to the material. If material is not included in the article's Creative Commons licence and your intended use is not permitted by statutory regulation or exceeds the permitted use, you will need to obtain permission directly from the copyright holder.

To view a copy of this licence, visit http://creativecommons.org/licenses/by/4.0/

\section{References}

Alanis Ruiz C, van Hooff T, Blocken B, Heijst GJF (2018). CFD analysis of the effect of pressure gradients on the separation efficiency of a generic air curtain. In: Proceedings of Roomvent\&Ventilation (REHVA 2018), Helsinki, Finland, pp. 241-246.

ANSYS (2013). ANSYS Fluent 15.0. User's Guide. ANSYS Inc.

Van Belleghem M, Verhaeghe G, T'Joen C, Huisseune H, De Jaeger P, De Paepe M (2012). Heat transfer through vertically downwardblowing single-jet air curtains for cold rooms. Heat Transfer Engineering, 33: 1196-1206.

Blocken B (2015). Computational Fluid Dynamics for urban physics: Importance, scales, possibilities, limitations and ten tips and tricks towards accurate and reliable simulations. Building and Environment, 91: 219-245.

Blocken B (2018). LES over RANS in building simulation for outdoor and indoor applications: a foregone conclusion? Building Simulation, 11: 821-870.

Casey M, Wintergerste T (2000). ERCOFTAC best practice guidelines: ERCOFTAC special interest group on "quality and trust in industrial CFD” (Version 1). European Research Community on Flow, Turbulence and Combustion.

Chen J-K, Huang RF, Hung W-L (2013). Flow and leakage characteristics of a sashless inclined air-curtain (sIAC) fume hood containing tall pollutant-generation tanks. Journal of Occupational and Environmental Hygiene, 10: 694-704.

Costa JJ, Oliveira LA, Silva MCG (2006). Energy savings by aerodynamic sealing with a downward-blowing plane air curtain-A numerical approach. Energy and Buildings, 38: 1182-1193.

Craft TJ, Graham LJW, Launder BE (1993). Impinging jet studies for turbulence model assessment-II. An examination of the performance of four turbulence models. International Journal of Heat and Mass Transfer, 36: 2685-2697.

Deo RC, Mi J, Nathan GJ (2007a). The influence of nozzle-exit geometric profile on statistical properties of a turbulent plane jet. Experimental Thermal and Fluid Science, 32: 545-559.

Deo RC, Mi J, Nathan GJ (2007b). The influence of nozzle aspect ratio on plane jets. Experimental Thermal and Fluid Science, 31: 825-838.

Dutta R, Dewan A, Srinivasan B (2013). Comparison of various integration to wall (ITW) RANS models for predicting turbulent slot jet impingement heat transfer. International Journal of Heat and Mass Transfer, 65: 750-764.

Foster AM, Swain MJ, Barrett R, D'Agaro P, James SJ (2006). Effectiveness and optimum jet velocity for a plane jet air curtain used to restrict cold room infiltration. International Journal of Refrigeration, 29: 692-699.

Frank D, Linden PF (2014). The effectiveness of an air curtain in the doorway of a ventilated building. Journal of Fluid Mechanics, 756: 130-164.

Frank D, Linden PF (2015). The effects of an opposing buoyancy force on the performance of an air curtain in the doorway of a building. Energy and Buildings, 96: 20-29.

Gonçalves JC, Costa JJ, Lopes AMG (2019). Parametric study on the performance of an air curtain based on CFD simulations - New proposal for automatic operation. Journal of Wind Engineering and Industrial Aerodynamics, 193: 103951.

Goubran S, Qi D, Saleh WF, Wang LL, Zmeureanu R (2016). Experimental study on the flow characteristics of air curtains at building entrances. Building and Environment, 105: 225-235.

Hayes FC, Stoecker WF (1969a). Heat transfer characteristics of the air curtain. ASHRAE Transactions, 75(2): 153-167.

Hayes FC, Stoecker WF (1969b). Design data for air curtains. ASHRAE Transactions, 75(2): 168-180.

Howell RH, Shibata M (1980). Optimum heat transfer through turbulent recirculated plane air curtains. ASHRAE Transactions, 86(1): 188-200.

Isman MK, Pulat E, Etemoglu AB, Can M (2008). Numerical investigation of turbulent impinging jet cooling of a constant heat flux surface. Numerical Heat Transfer, Part A: Applications, 53: 1109-1132.

Jaramillo JE, Pérez-Segarra CD, Rodriguez I, Oliva A (2008). Numerical study of plane and round impinging jets using RANS models. Numerical Heat Transfer, Part B: Fundamentals, 54: 213-237.

Khayrullina A, van Hooff T, Blocken B, van Heijst GJF (2017). PIV measurements of isothermal plane turbulent impinging jets at moderate Reynolds numbers. Experiments in Fluids, 58: 31.

Khayrullina A, van Hooff T, Blocken B, van Heijst G (2019). Validation of steady RANS modelling of isothermal plane turbulent impinging jets at moderate Reynolds numbers. European Journal of Mechanics, B/Fluids, 75: 228-243.

Koseoglu MF, Baskaya S (2010). The role of jet inlet geometry in impinging jet heat transfer, modeling and experiments. International Journal of Thermal Sciences, 49: 1417-1426.

Moureh J, Yataghene M (2016). Numerical and experimental study of airflow patterns and global exchanges through an air curtain subjected to external lateral flow. Experimental Thermal and Fluid Science, 74: 308-323.

Nielsen PV, Allard F, Awbi HB, Davidson L, Schälin A (2007). Computational fluid dynamics in ventilation design REHVA guidebook No 10. International Journal of Ventilation, 6: 291-294.

Nino E, Fasanella R, Di Tommaso RM (2011). Submerged rectangular air jets as a particulate barrier. Building and Environment, 46: 2375-2386.

Park TH, Choi HG, Yoo JY, Kim SJ (2003). Streamline upwind numerical simulation of two-dimensional confined impinging slot jets. International Journal of Heat and Mass Transfer, 46: 251-262.

Rajaratnam N (1976). Turbulent Jets. New York: Elsevier Scientific. 
Rhea S, Bini M, Fairweather M, Jones WP (2009). RANS modelling and LES of a single-phase, impinging plane jet. Computers \& Chemical Engineering, 33: 1344-1353.

Roache PJ (1994). Perspective: A method for uniform reporting of grid refinement studies. Journal of Fluids Engineering, 116: 405-413.

Roache PJ (1997). Quantification of uncertainty in computational fluid dynamics. Annual Review of Fluid Mechanics, 29: 123-160.

Schatzmann M, Olesen H, Franke J (2010). COST 732 Model Evaluation Case Studies: Approach and Results, COST Action.

Sharif MAR, Mothe KK (2009). Evaluation of turbulence models in the prediction of heat transfer due to slot jet impingement on plane and concave surfaces. Numerical Heat Transfer, Part B: Fundamentals, 55: 273-294.

Shih Y-C, Yang A-S, Lu C-W (2011). Using air curtain to control pollutant spreading for emergency management in a cleanroom. Building and Environment, 46: 1104-1114.

Sirén K (2003). Technical dimensioning of a vertically upwards blowing air curtain-part I. Energy and Buildings, 35: 681-695.

Tominaga Y, Stathopoulos T (2007). Turbulent Schmidt numbers for CFD analysis with various types of flowfield. Atmospheric Environment, 41: 8091-8099.
Valkeapää A, Sirén K, Raappana I (2006). Air leakage through horizontal air curtains-An experimental study. In: Proceedings of the 8th International conference (VENT 2006). Chicago, USA.

Wang LL, Zhong Z (2014). An approach to determine infiltration characteristics of building entrance equipped with air curtains. Energy and Buildings, 75: 312-320.

Wolfshtein M (1969). The velocity and temperature distribution in one-dimensional flow with turbulence augmentation and pressure gradient. International Journal of Heat and Mass Transfer, 12: 301-318.

Yakhot V, Orszag SA, Thangam S, Gatski TB, Speziale CG (1992). Development of turbulence models for shear flows by a double expansion technique. Physics of Fluids A: Fluid Dynamics, 4: 1510-1520.

Yang S, Alrawashdeh H, Zhang C, Qi D, Wang LL, Stathopoulos T (2019). Wind effects on air curtain performance at building entrances. Building and Environment, 151: 75-87.

Zhai ZJ, Osborne AL (2013). Simulation-based feasibility study of improved air conditioning systems for hospital operating room. Frontiers of Architectural Research, 2: 468-475. 